\title{
On the Floer homology of plumbed three-manifolds
}

\author{
Peter Ozsváth \\ Zoltán Szabó \\ Department of Mathematics, Columbia University \\ New York 10027, USA \\ and \\ Department of Mathematics, Princeton University \\ New Jersey 08540, USA \\ Email: petero@math.columbia.edu and szabo@math.princeton.edu
}

\begin{abstract}
We calculate the Heegaard Floer homologies for three-manifolds obtained by plumbings of spheres specified by certain graphs. Our class of graphs is sufficiently large to describe, for example, all Seifert fibered rational homology spheres. These calculations can be used to determine also these groups for other three-manifolds, including the product of a circle with a genus two surface.
\end{abstract}

\section{AMS Classification numbers Primary: 57R58}

Secondary: 57M27, 53D40, 57N12

Keywords: Plumbing manifolds, Seifert fibered spaces, Floer homology

Proposed: Robion Kirby

Seconded: Tomasz Mrowka, Yasha Eliashberg
Received: 15 April 2002

Revised: 28 January 2003

(c) Geometry $8 \mathcal{G}$ Topology Publications 


\section{Introduction}

In [13], we defined Heegaard Floer homology invariants for closed, oriented three-manifolds. In [11], we defined invariants for cobordisms between threemanifolds, and consequently also for smooth, closed four-manifolds. The resulting package has many properties of a topological quantum field theory, and moreover it is closely related to its gauge-theoretic counterparts, DonaldsonFloer (see [1]) and Seiberg-Witten theory (see [17], [9], [8]).

In particular, the three-manifold invariants are a fundamental stepping-stone in the definition and computation of the four-manifold invariants. Moreover, many four-dimensional aspects of the three-manifold $Y$ are reflected in its Heegaard Floer homology, including obstructions to embedding the three-manifold in a symplectic four-manifold (cf. [14]) and also restrictions on the intersection forms of smooth four-manifolds which bound $Y$ (see [15], compare [4]).

Whereas ingredients in the Heegaard Floer homology are more combinatorial in flavor than the corresponding gauge theory ingredients, the definition still involves a fundamentally analytical object: holomorphic disks in the symmetric product of a Riemann surface. Our aim here is to give a combinatorial formulation of these groups for a class of three-manifolds which are obtained by certain plumbing diagrams. Indeed, this class is large enough to describe, for example, all Seifert fibered rational homology spheres. The answer we describe can be read off from the plumbing tree. Moreover, this answer contains, as a by-product, all of the relative invariants of the four-manifold obtained from the plumbing description. It is interesting to compare these calculations with their corresponding analogues in instanton Floer homology (see for example [3], [7]) and Seiberg-Witten theory (see for example [10]). Note that, for many of the three-manifolds studied in this paper, the corresponding instanton Floer homology and Seiberg-Witten theory remain elusive.

Applications of these calculations include, as we have mentioned, non-embedding theorems for certain of these three-manifolds in symplectic four-manifolds, cf. [14]. As another application, we show that the four-manifold obtained from the plumbing description has, in some sense, a maximally exotic intersection form, as measured by the lengths of characteristic vectors, cf. Corollary 1.6 below. The computations in this paper also play a major role in [16], where we give constraints on knots in the three-sphere which admit Seifert fibered surgeries. A final application described here gives calculations of the Heegaard Floer homology for some other three-manifolds, including the product of a circle with a surface of genus two. This latter calculation is used to shed some light on the structure of Heegaard Floer homology for more complicated three-manifolds. 
With this motivation in hand, we describe the family of three-manifolds studied in this paper; but first, we give some preliminaries.

We call a weighted graph $G$ a graph equipped with an integer-valued function $m$ on its vertices. A weighted graph gives rise to a four-manifold with boundary $X(G)$ which is obtained by plumbing together a collection of disk bundles over the two-sphere (indexed by vertices of $G$ ), so that the Euler number of the sphere bundle corresponding to the vertex $v$ is given by its multiplicity $m(v)$. The sphere belonging to $v$ is plumbed to the sphere belonging to $w$ precisely when the two are connected by an edge. Let $Y(G)$ be the oriented threemanifold which is the boundary of $X(G)$.

For $X=X(G)$, the group $H_{2}(X ; \mathbb{Z})$ is the lattice freely spanned by the vertices of $G$, and the intersection form on $H_{2}(X ; \mathbb{Z})$ is given by the graph as follows. For a vertex $v$ of $G$, let $[v] \in H_{2}(X ; \mathbb{Z})$ denote the corresponding homology class. Then, for each vertex $[v] \cdot[v]=m(v)$, and for each pair of distinct vertices $v$ and $w,[v] \cdot[w]$ is one if $v$ and $w$ are connected by an edge, and zero otherwise.

Definition 1.1 A weighted graph is said to be a negative-definite graph if:

- $G$ is a disjoint union of trees

- the intersection form associated to $G$ is negative definite.

The degree of a vertex $v \in \operatorname{Vert}(G)$, denoted $d(v)$, is the number of edges which contain $v$. A vertex $v \in \operatorname{Vert}(G)$ is said to be a bad vertex of the weighted graph if

$$
m(v)>-d(v) .
$$

In this paper, we will be primarily concerned with negative-definite graphs with at most one bad vertex.

Note that any Seifert fibered rational homology sphere (with at least one orientation) can be realized from a negative-definite graph which is star-like (i.e. is a connected graph with at most one vertex with degree $>2$ ), so that if $v$ is a vertex with degree $d(v) \leq 2$, then $m(v) \leq-2$ (see for example [6]). In particular, this is a negative-definite graph with at most one bad vertex.

Our goal here is to give an algebraic description of the Heegaard Floer homology groups $H F^{+}(-Y(G))$. Recall that the Heegaard Floer homology groups come in a package, $H F^{+}, H F^{-}, H F^{\infty}$ and $\widehat{H F}$ which are all closely related. However, for a rational homology three-sphere, all of the information can be extracted from $\mathrm{HF}^{+}$. Recall that this group is in general a module over the ring 
$\mathbb{Z}[U]$, where $U$ lowers degree by two, and every element in $H^{+}$is annihilated by a sufficiently large power of $U$.

As a starting point, let $\mathcal{T}_{0}^{+}$denote the graded $\mathbb{Z}[U]$-module which is the quotient of $\mathbb{Z}\left[U, U^{-1}\right]$ by the submodule $U \cdot \mathbb{Z}[U]$. This module is graded so that the element $U^{-d}$ (for $d \geq 0$ ) is supported in degree $2 d$. Recall that

$$
H F^{+}\left(S^{3}\right) \cong \mathcal{T}_{0}^{+} .
$$

Let $\operatorname{Char}(G)$ denote the set of characteristic vectors for the intersection form. Let

$$
\mathbb{H}^{+}(G) \subset \operatorname{Hom}\left(\operatorname{Char}(G), \mathcal{T}_{0}^{+}\right)
$$

denote the set of functions with finite support and which satisfy the following "adjunction relations" for all characteristic vectors $K$ and vertices $v$. Let

$$
2 n=\langle K, v\rangle+v \cdot v .
$$

If $n \geq 0$, then we require that

$$
U^{n} \cdot \phi(K+2 \mathrm{PD}[v])=\phi(K),
$$

while if $n \leq 0$, then

$$
\phi(K+2 \mathrm{PD}[v])=U^{-n} \cdot \phi(K) .
$$

We can decompose $\mathbb{H}^{+}(G)$ according to $\operatorname{Spin}^{c}$ structures over $Y$. Note first that the first Chern class gives an identification of the set of $\operatorname{Spin}^{c}$ structures over $X=X(G)$ with the set of characteristic vectors $\operatorname{Char}(G)$. Observe that the image of $H^{2}(X, \partial X ; \mathbb{Z})$ in $H^{2}(W ; \mathbb{Z})$ is spanned by the Poincaré duals of the spheres corresponding to the vertices. Using the restriction to boundary, it is easy to see that the set of $\operatorname{Spin}^{c}$ structures over $Y$ is identified with the set of $2 H^{2}(X, \partial X ; \mathbb{Z})$-orbits in $\operatorname{Char}(G)$.

Fix a $\operatorname{Spin}^{c}$ structure $\mathfrak{t}$ over $Y$. Let $\operatorname{Char}_{\mathfrak{t}}(G)$ denote the set of characteristic vectors for $X$ which are first Chern classes of $\operatorname{Spin}^{c}$ structures $\mathfrak{s}$ whose restriction to the boundary is $\mathfrak{t}$. Similarly, we let

$$
\mathbb{H}^{+}(G, \mathfrak{t}) \subset \mathbb{H}^{+}(G)
$$

be the subset of maps which are supported on the subset of characteristic vectors $\operatorname{Char}_{\mathfrak{t}}(G) \subset \operatorname{Char}(G)$. We have a direct sum splitting:

$$
\mathbb{H}^{+}(G) \cong \bigoplus_{\mathfrak{t} \in \operatorname{Spin}^{c}(Y)} \mathbb{H}^{+}(G, \mathfrak{t}) .
$$


We can also introduce a grading on $\mathbb{H}^{+}(G)$ as follows. We say that an element $\phi \in \mathbb{H}^{+}(G)$ is homogeneous of degree $d$ if for each characteristic vector $K$ with $\phi(K) \neq 0, \phi(K) \in \mathcal{T}_{0}^{+}$is a homogeneous element with:

$$
\operatorname{deg}(\phi(K))-\left(\frac{K^{2}+|G|}{4}\right)=d .
$$

Our main result is the following identification of $\mathrm{HF}^{+}(-Y(G))$ in terms of combinatorics of the plumbing diagram:

Theorem 1.2 Let $G$ be a negative-definite weighted graph with at most one bad vertex, in the sense of Definition 1.1. Then, for each $\operatorname{Spin}^{c}$ structure $\mathfrak{t}$ over $-Y(G)$, there is an isomorphism of graded $\mathbb{Z}[U]$ modules,

$$
H F^{+}(-Y(G), \mathfrak{t}) \cong \mathbb{H}^{+}(G, \mathfrak{t}) .
$$

Remark 1.3 It is a straightforward matter to determine $\mathrm{HF}^{+}(Y(G), \mathfrak{t})$ from $H F^{+}(-Y(G), \mathfrak{t})$, cf. Section 2 of $[12]$.

In the statement of the above theorem, the grading on $H F^{+}(-Y(G), \mathfrak{t})$ is the absolute $\mathbb{Q}$-grading defined in [11] and studied in [15]. Recall that when $-Y(G)$ is an integral homology sphere, this absolute grading takes values in $\mathbb{Z}$.

As a qualitative remark, it is perhaps worth pointing out the following corollary (compare [3]). To state it, recall that there is an absolute $\mathbb{Z} / 2 \mathbb{Z}$-grading on $H F^{+}(Y, \mathfrak{t})$ which, for rational homology three-spheres, is determined by the following criterion. A homogeneous element $\xi$ is even with respect to this grading if there is a non-zero homogeneous element of $\xi_{0} \in H F^{\infty}(Y, \mathfrak{t})$ with the property that

$$
\operatorname{deg}(\xi)-\operatorname{deg}\left(\xi_{0}\right) \equiv 0 \quad(\bmod 2) .
$$

When $Y$ is an integral homology sphere, this notion coincides with the parity of the ( $\mathbb{Z}$-)grading of $\xi$.

Corollary 1.4 If $G$ is a negative-definite graph with at most one bad vertex, then all elements of $\mathrm{HF}^{+}(-Y(G), \mathfrak{t})$ have even $\mathbb{Z} / 2 \mathbb{Z}$ grading.

Proof This follows immediately from Theorem 1.2 and the definition of the absolute gradings: $\phi(K) \in \mathcal{T}_{0}^{+}$, and the latter module is supported only in even degrees. 
This underscores the importance of the hypothesis on the graph. For example, if $Y$ is the Brieskorn homology sphere $\Sigma(2,3,7)$ (which can be thought of as (-1)surgery on the right-handed trefoil knot) then it follows easily from the Künneth formula for connected sums (Theorem 6.2 of [12]) that $\mathrm{HF}^{+}(-(Y \# Y))$ has elements of both parities. On the other hand, $Y \# Y$ admits a plumbing description as a negative-definite disconnected graph with two bad points. For an example belonging to a connected graph, one can take -1 surgery on the connected sum of two right-handed trefoil knots in $S^{3}$, see Proposition 4.2 and Remark 4.3 below. The methods for obtaining Theorem 1.2 do, however, give information on the Floer homology groups of the three-manifolds obtained from these plumbing diagrams as well, see Theorem 2.2 below.

Theorem 2.1 also has the following corollary. For the purpose of this corollary, recall that in [15], we defined an invariant $d(Y, \mathfrak{t})$ associated to an oriented, rational homology three-sphere $Y$ equipped with a $\operatorname{Spin}^{c}$ structure $\mathfrak{t}$. This invariant takes values in $\mathbb{Q}$, The importance of $d(Y, \mathfrak{t})$ is shown by the fact that it gives a bound on the exoticness of the intersection form for any smooth, definite four-manifold which bounds $Y$. Specifically, if $Y$ is a rational homology three-sphere equipped with a $\operatorname{Spin}^{c}$ structure $\mathfrak{t}$, then if $W$ is an oriented fourmanifold with negative-definite intersection form, and $\mathfrak{s}$ is any $\operatorname{Spin}^{c}$ structure over $W$ whose restriction to $Y$ is $\mathfrak{t}$, then Theorem 9.6 of [15] establishes the inequality

$$
c_{1}(\mathfrak{s})^{2}+\operatorname{rk} H^{2}(X ; \mathbb{Z}) \leq 4 d(Y, \mathfrak{t}) .
$$

Compare also the gauge-theoretic version of Frøyshov, [4] and [5]. (For the relationship between diagonalizability of definite, unimodular forms $Q$ and the maximal value, over all characteristic vectors $K$ for $Q$, of the quantity $K^{2}+$ rk, see [2].) We have the following consequence of Theorem 2.1 (which, in the case where $G$ has two bad points, follows from Theorem 2.2):

Corollary 1.5 Let $G$ be a negative-definite graph with at most two bad points, and fix a $\operatorname{Spin}^{c}$ structure $\mathfrak{t}$ over $Y$. Then,

$$
d(Y(G), \mathfrak{t})=\max _{\left\{K \in \operatorname{Char}_{\mathfrak{t}}(G)\right\}} \frac{K^{2}+|G|}{4} .
$$

The above result gives a practical calculation of $d(Y, \mathfrak{t}):$ for a given $\mathfrak{t} \in \operatorname{Spin}^{c}(Y)$, it is easy to see that the maximum of $\frac{K^{2}+|G|}{4}$ is always achieved among the finitely many characteristic vectors $K \in \operatorname{Char}_{\mathfrak{t}}(G)$ with

$$
|K \cdot v| \leq|m(v)| \text {. }
$$


(A smaller set containing these minimal vectors is described in Proposition 3.2 below.)

Inequality (4), combined with Corollary 1.5, immediately gives the following:

Corollary 1.6 Let $G$ be a negative-definite graph with at most two bad points, and fix a Spin ${ }^{c}$ structure $\mathfrak{t}$ over $Y$. Then, for each smooth, compact, oriented four-manifold $X$ with negative intersection form which bounds $Y$, and for each $\operatorname{Spin}^{c}$ structure $\mathfrak{s} \in \operatorname{Spin}^{c}(X)$ with $\mathfrak{s} \mid Y=\mathfrak{t}$, we have that

$$
c_{1}(\mathfrak{s})^{2}+\operatorname{rk}\left(H^{2}(X ; \mathbb{Z})\right) \leq \max _{\left\{K \in \operatorname{Char}_{\mathfrak{t}}(G)\right\}} K^{2}+|G| .
$$

The above results are proved in Section 2. In Section 3 we give some sample calculations. In Section 4, we use these techniques as a starting-point for another calculation: the calculation of $H F^{+}\left(S^{1} \times \Sigma_{2}\right)$ (cf. Theorem 4.9 below).

We end the paper with some speculations based on this latter result. Specifically, recall that we defined in [13] and [12] a group $H F^{\infty}$ which captures the behaviour of $\mathrm{HF}^{+}$in all sufficiently large degrees. When the three-manifold has $b_{1}(Y)<3, H F^{\infty}$ is determined by $b_{1}(Y)$. It remains an interesting question to determine $H F^{\infty}$ for arbitrary three-manifolds. We conclude this paper with a conjecture relating $H F^{\infty}(Y)$ with the cohomology ring of $Y$.

Acknowledgements We would like to thank the referee for a careful reading of the manuscript and some very useful comments.

PSO was supported by NSF grant number DMS 9971950 and a Sloan Research Fellowship; ZSz was supported by NSF grant number DMS 0107792 and a Packard Fellowship.

\section{Proof of Theorem 1.2}

In the present section, we state a more precise version of Theorem 1.2, and give a proof. For the more precise statement, we need the following notions.

First, to postpone a discussion of signs which might obscure matters, we work over the field with two elements $\mathbb{F}=\mathbb{Z} / 2 \mathbb{Z}$ for the rest of the subsection, returning to a sign-refinement which allows us to work over $\mathbb{Z}$ in Subsection 2.1. Thus, unless it is explicitly stated otherwise, all Floer homology groups in this subsection are meant to be taken with $\mathbb{F}$ coefficients (which we suppress 
from the notation). In particular, with these conventions, $\mathcal{T}_{0}^{+}$now denotes the quotient of $\mathbb{F}\left[U, U^{-1}\right]$ by the submodule $U \cdot \mathbb{F}[U]$.

We define a map

$$
T^{+}: H F^{+}(-Y(G)) \longrightarrow \mathbb{H}^{+}(G)
$$

as follows. The plumbing diagram can be viewed as giving a cobordism $W(G)$ from $S^{3}$ to the three-manifold $Y(G)$ (i.e. this is the four-manifold obtained by deleting a ball from the four-manifold $X(G)$ considered in the introduction) or, equivalently, a cobordism from $-Y(G)$ to $S^{3}$. Now let

$$
T^{+}(\xi): \operatorname{Char}(G) \longrightarrow \mathcal{T}_{0}^{+}
$$

be the map given by

$$
T^{+}(\xi)(K)=F_{W(G), \mathfrak{s}}^{+}(\xi) \in H F^{+}\left(S^{3}\right)=\mathcal{T}_{0}^{+},
$$

where $\mathfrak{s} \in \operatorname{Spin}^{c}(W(G))$ is the $\operatorname{Spin}^{c}$ structure whose first Chern class is $K$, and $F_{W(G), \mathfrak{s}}^{+}$denotes the four-dimensional cobodism invariant defined in [11].

Theorem 2.1 Let $G$ be a negative-definite graph with at most one bad vertex. Then, $T^{+}$induces a grading-preserving isomorphism:

$$
\mathbb{H}^{+}(G, \mathfrak{t}) \cong H F^{+}(-Y(G), \mathfrak{t}) .
$$

These techniques can be pushed further to obtain the following:

Theorem 2.2 Let $G$ be a negative-definite graph with at most two bad vertices. Then, $T^{+}$produces an isomorphism of graded $\mathbb{Z}[U]$-modules

$$
\mathbb{H}^{+}(G, \mathfrak{t}) \cong H F_{\mathrm{ev}}^{+}(-Y(G), \mathfrak{t}),
$$

where $\mathrm{HF}_{\mathrm{ev}}^{+}$denotes the part of $\mathrm{HF}^{+}$with even parity (using the absolute $\mathbb{Z} / 2 \mathbb{Z}$ grading).

In practice, it is sometimes easier to think about $\mathbb{H}^{+}(G)$ from the following dual point of view. We let $\mathbb{K}^{+}(G)$ denote the equivalence classes in $\mathbb{Z}^{\geq 0} \times \operatorname{Char}(G)$ (where we write a pair $m$ and $K$ as $U^{m} \otimes K$ ) under the following equivalence relation. Let $v$ be a vertex and let

$$
2 n=\langle K, v\rangle+v \cdot v .
$$

If $n \geq 0$, then:

$$
U^{n+m} \otimes(K+2 \mathrm{PD}[v]) \sim U^{m} \otimes K,
$$

while if $n \leq 0$, then

$$
U^{m} \otimes(K+2 \operatorname{PD}[v]) \sim U^{m-n} \otimes K .
$$


Given a function

$$
\phi: \operatorname{Char}(G) \longrightarrow \mathcal{T}_{0}^{+},
$$

there is an induced map

$$
\widetilde{\phi}: \mathbb{Z}^{\geq 0} \times \operatorname{Char}(G) \longrightarrow \mathcal{T}_{0}^{+}
$$

defined by

$$
\widetilde{\phi}\left(U^{n} \otimes K\right)=U^{n} \cdot \phi(K) .
$$

Clearly, the set of finitely-supported functions $\phi: \operatorname{Char}(G) \longrightarrow \mathcal{T}_{0}^{+}$whose induced map $\widetilde{\phi}$ descends to $\mathbb{K}^{+}(G)$ is precisely $\mathbb{H}^{+}(G)$.

Lemma 2.3 Let $\mathcal{B}_{n}$ denote the set of characteristic vectors

$$
\mathcal{B}_{n}=\{K \in \operatorname{Char}(G)|\forall v \in G,|\langle K, v\rangle \mid \leq-m(v)+2 n\} .
$$

The quotient map induces a surjection from

$$
\bigcup_{i=0}^{n} U^{i} \otimes \mathcal{B}_{n-i}
$$

onto the quotient space

$$
\frac{\mathbb{K}^{+}(G)}{\mathbb{Z}^{>n} \times \operatorname{Char}(G)} .
$$

In turn, we have an identification

$$
\left(\operatorname{Ker} U^{n+1} \subset \mathbb{H}^{+}(G ; \mathbb{F})\right) \cong \operatorname{Hom}\left(\frac{\mathbb{K}^{+}(G)}{\mathbb{Z}^{>n} \times \operatorname{Char}(G)}, \mathbb{F}\right)
$$

(i.e. the right-hand-side consists of maps from $\mathbb{K}^{+}(G)$ to $\mathbb{F}$ which vanish on the equivalence classes which contain representatives of the form $U^{m} \otimes K^{\prime}$ with $m>n$ ) and, indeed,

$$
\left(\operatorname{Ker} U^{n+1} \subset \mathbb{H}^{+}(G ; \mathbb{Z})\right) \cong \operatorname{Hom}\left(\frac{\mathbb{K}^{+}(G)}{\mathbb{Z}^{>n} \times \operatorname{Char}(G)}, \mathbb{Z}\right) .
$$

Proof The surjectivity statement follows easily from the definition of the equivalence relation in $\mathbb{K}^{+}(G)$.

The duality map is the one sending

$$
\phi \times\left(U^{\ell} \otimes K\right) \mapsto\left(U^{\ell} \cdot \phi(K)\right)_{0}
$$

(i.e. taking the part in $\mathcal{T}_{0}^{+}$which lies in degree zero). This obviously induces a map

$$
\operatorname{Ker} U^{n+1} \longrightarrow \operatorname{Hom}\left(\frac{\mathbb{K}^{+}(G)}{\mathbb{Z}^{>n} \times \operatorname{Char}(G)}, \mathbb{Z}\right)
$$


This map is injective, since if $\phi(K) \in \mathcal{T}_{0}^{+}$is an element with

$$
\left(U^{\ell} \cdot \phi(K)\right)_{0} \equiv 0
$$

for all $\ell>n$, then clearly $U^{n+1} \cdot \phi(K)=0$. To see that the map is surjective, observe that if

$$
\tau \in \operatorname{Hom}\left(\frac{\mathbb{K}^{+}(G)}{\mathbb{Z}^{>n} \times \operatorname{Char}(G)}, \mathbb{Z}\right)
$$

is an arbitrary element, we can define a map

$$
\phi(K)=\sum_{\ell=0}^{n} \tau\left(U^{\ell} \otimes K\right) \cdot U^{-\ell}
$$

whose degree zero part is $\tau$. Clearly, $\phi \in \mathbb{H}^{+}(G)$, and $U^{n+1} \cdot \phi=0$.

We now set up some properties of $\mathbb{H}^{+}(G, \mathfrak{t})$ with a view towards proving Theorem 2.1.

Proposition 2.4 The map $T^{+}$induces an $\mathbb{F}[U]$-equivariant, degree-preserving map from $\mathrm{HF}^{+}(-Y(G), \mathfrak{t})$ to $\operatorname{Hom}\left(\operatorname{Char}_{\mathfrak{t}}(G), \mathcal{T}_{0}^{+}\right)$whose image lies in

$$
\mathbb{H}^{+}(G, \mathfrak{t}) \subset \operatorname{Hom}\left(\operatorname{Char}_{\mathfrak{t}}(G), \mathcal{T}_{0}^{+}\right) .
$$

Proof The map $T^{+}$lands inside $\operatorname{Hom}\left(\operatorname{Char}_{\mathfrak{t}}(G), \mathcal{T}_{0}^{+}\right)$with finite support, according to general finiteness properties of the maps on $\mathrm{HF}^{+}$induced by cobordisms (cf. Theorem 3.3 of [11]). Alternatively, this finiteness follows from the degree shift formula for maps induced by cobordisms, Theorem 7.1 of [11], which also shows that $T^{+}$is degree-preserving. The fact that $T^{+}$lands in the subset of $\operatorname{Hom}\left(\operatorname{Char}_{\mathfrak{t}}(G), \mathcal{T}_{0}^{+}\right)$satisfying the adjunction relation (Equation (1) or (2) as appropriate) defining $\mathbb{H}^{+}(G)$ is proved in Theorem 3.1 of [14] (where, actually, relations are established for oriented, embedded surfaces of arbitrary genus).

If $G$ is a weighted graph with a distinguished vertex $v \in \operatorname{Vert}(G)$, we let $G^{\prime}(v)$ be a new graph formed by introducing one new vertex $e$ labelled with weight -1 , and connected to only one other vertex, $v$. Moreover, we let $G_{+1}(v)$ denote the weighted graph whose underlying graph agrees with $G$, but whose weight at $v$ is increased by one (and the weight stays the same for all other vertices). The two three-manifolds $Y\left(G^{\prime}(v)\right)$ and $Y\left(G_{+1}(v)\right)$ are clearly diffeomorphic; in fact, we have the following: 
Proposition 2.5 Let $G^{\prime}(v)$ be the graph obtained from $G$ as above. Then, there is a grading-preserving isomorphism

$$
R: \mathbb{H}^{+}\left(G^{\prime}(v)\right) \rightarrow \mathbb{H}^{+}\left(G_{+1}(v)\right)
$$

Moreover, this map is natural with $T^{+}$in the sense that:

$$
\begin{array}{ccc}
H F^{+}\left(-Y\left(G^{\prime}(v)\right)\right) & \Theta & H F^{+}\left(-Y\left(G_{+1}(v)\right)\right) \\
T_{G^{\prime}(v)}^{+} \downarrow & & T_{G_{+1}(v)}^{+} \downarrow \\
\mathbb{H}^{+}\left(G^{\prime}(v)\right) & \stackrel{R}{\longrightarrow} & \mathbb{H}^{+}\left(G_{+1}(v)\right),
\end{array}
$$

where map $\Theta$ is the isomorphism induced by the diffeomorphism of $Y\left(G^{\prime}(v)\right) \cong$ $Y\left(G_{+1}(v)\right)$.

Proof We construct $R$ in two steps. As a first step, let $G_{+1}(v) \cup f$ denote the disconnected graph consisting of the disjoint union of $G_{+1}(v)$ and a single vertex $f$ with multiplicity -1 . We have a map

$$
\operatorname{Char}\left(G_{+1}(v) \cup f\right) \cong \operatorname{Char}\left(G^{\prime}(v)\right)
$$

induced by a change of basis. It is easy to see that this map induces an isomorphism

$$
\mathbb{H}^{+}\left(G_{+1}(v) \cup f\right) \cong \mathbb{H}^{+}\left(G^{\prime}(v)\right) .
$$

Next, we define a map

$$
Q: \mathbb{H}^{+}\left(G_{+1}(v)\right) \longrightarrow \mathbb{H}^{+}\left(G_{+1}(v) \cup f\right)
$$

by the formula (where $m \geq 0$ )

$$
\begin{aligned}
Q(\phi)(K,(2 m+1)) & =U^{m(m+1) / 2} \cdot \phi(K), \\
Q(\phi)(K,-(2 m+1)) & =U^{m(m+1) / 2} \cdot \phi(K) .
\end{aligned}
$$

In the above notation, $(K, \ell)$ denotes the characteristic vector for $G_{+1}(v) \cup f$ whose restriction to $G_{+1}(v)$ is $K$ and whose evaluation on $f$ is $\ell$. The map $Q$, too, is clearly an isomorphism. We define $R$ to be the composition of the above isomorphisms.

To check commutativity of the diagram, observe that we have a diffeomorphism of four-manifolds $W\left(G^{\prime}(v)\right) \cong W\left(G_{+1}(v) \cup f\right)$, arising by sliding the circle corresponding to $v$ (in $G^{\prime}(v)$ ) over the circle $e$. By the handleslide invariance of the maps induced by cobordisms (cf. [11]) and the blow-up formula, we have the identification

$$
F_{W\left(G^{\prime}(v)\right), K^{\prime}}^{+}(\xi)=F_{W\left(G_{+1}(v) \cup f\right),(K, 1)}^{+}\left(\xi^{\prime}\right)=F_{W\left(G_{+1}(v)\right), K}^{+}\left(\xi^{\prime \prime}\right),
$$


where here $\xi^{\prime}$ and $\xi^{\prime \prime}$ are obtained from $\xi$ by equivalences of the Heegaard diagrams belonging to $-Y\left(G^{\prime}(v)\right),-Y\left(G_{+1}(v) \cup f\right)$, and $-Y\left(G_{+1}(v)\right)$, and $K^{\prime}$ is the characteristic vector of the $\operatorname{Spin}^{c}$ structure over $G^{\prime}(v)$ whose characteristic vector can be written as $(K, 1)$ under the change-of-basis corresponding to $W\left(G^{\prime}(v)\right) \cong W\left(G_{+1}(v) \cup f\right)$. Commutativity of the square now follows.

We begin with a few remarks on the case of a graph with no bad points. Recall that for such graphs, $H F_{\text {red }}^{+}(Y(G))=0$, as established in Theorem 7.1 of [14]. This follows easily from the following lemma, whose proof we include here for the reader's convenience:

Lemma 2.6 If $G$ is a negative-definite graph with no bad vertices, then $H_{1}(Y(G) ; \mathbb{Z})=\operatorname{rk} \widehat{H F}(Y(G))$.

Proof To show that $\left|H_{1}(Y(G) ; \mathbb{Z})\right|=\operatorname{rk} \widehat{H F}(Y(G))$, one shows that both of these numbers are additive in the sense that:

$$
N(G)=N\left(G_{+1}(v)\right)+N(G-v)
$$

(provided that $G_{+1}(v)$ also has no bad points) and they both satisfy the normalization that

$$
N(\text { empty graph })=1 .
$$

(Additivity of $\operatorname{rk} \widehat{H F}(Y(G))$ follows easily from the long exact sequence of $\widehat{H F}$; additivity of $\left|H_{1}(Y(G) ; \mathbb{Z})\right|$ is elementary.)

The equality of the two quantities now follows from induction, and the following observation: if $G$ is a graph with no bad vertices and $v$ is a leaf (i.e. a vertex with degree $d(v)=1$ ) with multiplicity -1 , then $G$ can be "blown down" to produce a graph with no bad vertices and one fewer vertex.

Lemma 2.7 Let $G$ be a graph which satisfies the inequality at each vertex $v$ :

$$
m(v)<-d(v) .
$$

Then, the rank of $\operatorname{Ker} U \subset \mathbb{H}^{+}(G)$ is the number of $\operatorname{Spin}^{c}$ structures over $Y$.

Proof In view of Lemma 2.3, the rank of $\operatorname{Ker} U$ is determined by the number of inequivalent characteristic vectors in $\mathcal{B}_{0}$, which are also not equivalent to elements in $\mathbb{Z}^{>0} \times \operatorname{Char}(G)$. Indeed, by subtracting off Poincaré duals of vertices as required, this is equal to the number of distinct characteristic vectors which 
are not equivalent to vectors of the form $U^{n} \otimes K^{\prime}$ with $n>0$ and which satisfy the inequality:

$$
m(v)+2 \leq K(v) \leq-m(v)
$$

at each vertex $v$. We call such a characteristic vector a short vector for $G$, and let $\mathcal{S}(G)$ denote the set of short vectors. The proposition, then, is equivalent to the statement that the number of vectors in $\mathcal{S}(G)$ agrees with the order of $H_{1}(Y(G) ; \mathbb{Z})$.

To this end, let $v$ be a leaf of $G$, and $w$ be a neighbor of $v$. We claim that

$$
|\mathcal{S}(G)|=-m(v)|\mathcal{S}(G-v)|-|\mathcal{S}(G-v-w)| .
$$

This equation implies the lemma by induction on the number of vertices in the graph, since $(-1)^{|G|} \operatorname{det}(G)$ (which counts the order of $H_{1}(Y(G) ; \mathbb{Z})$ and hence the number of $\operatorname{Spin}^{c}$ structures over $Y(G)$ ) clearly satisfies the same relation.

First, we claim that for each short vector $K$ for $G-v-w$, there is some constant $m(w)+2 \leq c(K)$ with the property that $(K, p)$ is a short vector for $G-v$ for all $m(w)+2 \leq p \leq c(K)$; and indeed, all the short vectors for $G-v$ arise in this manner. Then, Equation (10) follows from the following claim: the set of short vectors for $G$ whose restriction to $G-v-w$ is $K$ is given by:

$$
\left(\bigcup_{\substack{m(w)+2 \leq p \leq c(K), m(v)+2 \leq i \leq-m(v)}}(K, p, i)\right)-(K, c(K),-m(v)) .
$$

To see the existence of $c(K)$ as above, we proceed as follows. A characteristic vector $L$ (for any negative-definite, weighted graph) which satisfies inequalities $m(v)+2 \leq L(v) \leq-m(v)$ at each vertex $v$ is equivalent to a vector of the form $U^{n} \otimes L^{\prime}$ with $n>0$ if and only if there is a subset $\left\{v_{1}, \ldots, v_{k}\right\}$ of vertices and an element $A=a_{1} \mathrm{PD}\left[v_{1}\right]+\cdots+a_{k} \mathrm{PD}\left[v_{k}\right]$ with all $a_{i}>0$, so that

$$
L \cdot A+A \cdot A>0
$$

(see Proposition 3.2 below). Moreover, it follows easily from this same discussion that if $G$ satisfies Inequality (8) at each vertex, then we can arrange for all the $a_{i} \in\{0,1\}$. We use this to conclude that if $K$ is a short vector for $G-v-w$, then $L=(K, m(v)+2)$ is a short vector for $G-v$ : if not, we would have an expression $A$ as above, and, since $K$ is short for $G-v-w$, $w \in\left\{v_{1}, \ldots, v_{k}\right\}$, so we write $A=A_{0}+\mathrm{PD}[w]$. Let $j$ be the number of spheres appearing in the expression for $A_{0}$ (with non-zero multiplicity) which meet $w$. Then,

$$
L \cdot A+A \cdot A=K \cdot A_{0}+A_{0} \cdot A_{0}+2 m(w)+2+2 j .
$$


Since $j \leq d(w)<-m(w)$, and $K \in \mathcal{S}(G-v-w)$, it follows that $L \cdot A+A \cdot A<0$, contradicting Inequality (11).

The count of short vectors in $G$ with fixed restriction to $G-v-w$ (which establishes Equation (10)) proceeds similarly.

Suppose $G$ is a graph with a distinguished vertex $v$. Clearly, $Y(G)$ is obtained from $Y(G-v)$ by a single two-handle addition, and $Y\left(G^{\prime}(v)\right)$ is also obtained from $Y(G)$ by a single two-handle addition. These two-handle additions can be viewed as cobordisms from $-Y(G)$ to $-Y(G-v)$ and $-Y\left(G^{\prime}(v)\right)$ to $-Y(G)$ respectively. The induced maps fit into a short exact sequence, as follows:

Proposition 2.8 Suppose that $G_{+1}(v)$ is a negative-definite plumbing diagram, and suppose that $G-v$ contains no bad points. Then, there is a short exact sequence:

$$
0 \rightarrow H F^{+}\left(-Y\left(G^{\prime}(v)\right)\right) \stackrel{A^{+}}{\longrightarrow} H F^{+}(-Y(G)) \stackrel{B^{+}}{\longrightarrow} H F^{+}(-Y(G-v)) \rightarrow 0,
$$

where the maps $A^{+}$and $B^{+}$above are induced by the two-handle additions.

Proof Theorem 9.12 of [12] gives a long exact sequence

$$
\begin{aligned}
\cdots \longrightarrow H F^{+} & \left(-Y\left(G^{\prime}(v)\right)\right) \stackrel{A^{+}}{\longrightarrow} H F^{+}(-Y(G)) \\
& \stackrel{B^{+}}{\longrightarrow} H F^{+}(-Y(G-v)) \stackrel{C^{+}}{\longrightarrow} H F^{+}\left(-Y\left(G^{\prime}(v)\right)\right) \longrightarrow \cdots
\end{aligned}
$$

where the maps $A^{+}, B^{+}$, and $C^{+}$are induced by two-handle additions (in the sense of [11]).

The hypothesis that $G_{+1}(v)$ is negative-definite ensures that the two cobordisms inducing $A^{+}$and $B^{+}$above are both negative-definite; it follows that the third cobordism is not, and hence it induces the trivial map on $H F^{\infty}$ (cf. Lemma 8.2 of [11]). Since $G-v$ has no bad points, it follows easily from Lemma 2.6 that $H F_{\text {red }}^{+}(-Y(G-v))=0$. Thus, the map $C^{+}$is trivial.

Note that the above short exact sequence (together with the obvious induction on the graph) suffices to prove Corollary 1.4.

To prove Theorem 2.1, we compare the short exact sequence of Proposition 2.8 with a corresponding sequence for $\mathbb{H}^{+}$, defined as follows.

We write characteristic vectors for $G^{\prime}(v)$ as triples $(K, i, \ell)$, where $K$ is the restriction to $G-v$, and $i$ and $\ell$ denote the evaluations on the vertices $v$ and 
$f$ respectively (in particular, here $i \equiv m(v)(\bmod 2)$, and $\ell \equiv 1(\bmod 2))$. Similarly, we write characteristic vectors for $G$ as pairs $(K, i)$.

Given a finitely supported map $\phi \in \operatorname{Hom}\left(\operatorname{Char}\left(G^{\prime}(v)\right), \mathcal{T}_{0}^{+}\right)$, we let

$$
\mathbb{A}^{+}(\phi) \in \operatorname{Hom}\left(\operatorname{Char}(G), \mathcal{T}_{0}^{+}\right)
$$

be the map defined by

$$
\left\langle\mathbb{A}^{+}(\phi),(K, p)\right\rangle=\sum_{j=-\infty}^{+\infty} \phi(K, p, 2 j+1) .
$$

Similarly, given a finitely supported map $\phi \in \operatorname{Hom}\left(\operatorname{Char}(G), \mathcal{T}_{0}^{+}\right)$, we let

$$
\mathbb{B}^{+}(\phi) \in \operatorname{Hom}\left(\operatorname{Char}(G-v), \mathcal{T}_{0}^{+}\right)
$$

be the map defined by

$$
\left\langle\mathbb{B}^{+}(\phi), K\right\rangle=\sum_{i=-\infty}^{+\infty} \phi(K, 2 i+m(v)) .
$$

Lemma 2.9 The above formulas induce maps

$$
\mathbb{A}^{+}: \mathbb{H}^{+}\left(G^{\prime}(v)\right) \longrightarrow \mathbb{H}^{+}(G) \text { and } \mathbb{B}^{+}: \mathbb{H}^{+}(G) \longrightarrow \mathbb{H}^{+}(G-v) \text {. }
$$

Proof The proof is straightforward.

Next, we verify that these maps fit together with the maps of Proposition 2.8 as follows:

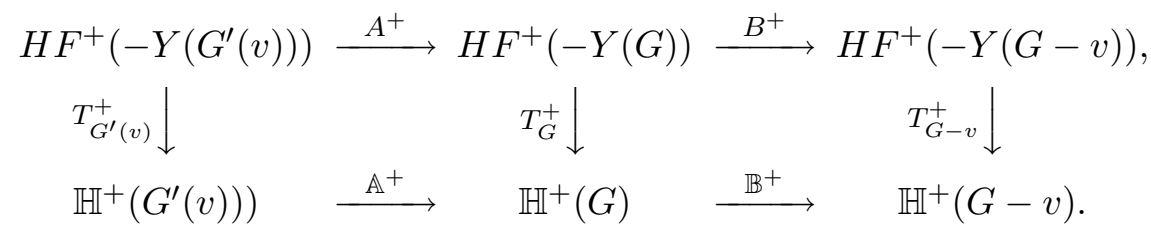

Lemma 2.10 Let $G$ be a graph with the property that $G^{\prime}(v)$ is negativedefinite. Then, the squares in Diagram (12) commute. Moreover, $\mathbb{A}^{+}$is injective, and

$$
\mathbb{B}^{+} \circ \mathbb{A}^{+}=0 \text {. }
$$

Proof Commutativity of the squares follows immediately from the naturality of the maps under composition of cobordisms. 
To verify the injectivity of $\mathbb{A}^{+}$, we proceed as follows. Note that the grading on $\mathcal{T}_{0}^{+}$induces a filtration in the natural way. Specifically, if $\xi \in \mathcal{T}_{0}^{+}$is a non-zero element, we can write

$$
\xi=\xi_{0}+\ldots+\xi_{n},
$$

where $\xi_{i}$ denotes the homogeneous component of $\xi$ in dimension $i$, so that $\xi_{n} \neq 0$. Then, we define $\mathcal{F}(\xi)=n$. By convention, $\mathcal{F}(0)=-\infty$.

Now, fix some non-zero $\phi \in \mathbb{H}^{+}\left(G^{\prime}(v)\right)$, and let $\mathcal{K}_{1} \subset \operatorname{Char}\left(G^{\prime}(v)\right)$ denote the set of characteristic vectors $K$ which maximize $\mathcal{F}(\phi(K)$ ) (amongst all characteristic vectors). Such a vector can be found since $\phi$ has finite support. Next, let $\mathcal{K}_{2} \subset \mathcal{K}_{1}$ denote the subset for which $\langle K, v\rangle$ is maximal. We claim that if $K \in \mathcal{K}_{2}$, then

$$
\mathbb{A}^{+}(\phi)(K \mid G) \neq 0 .
$$

In fact, letting $\mathcal{E}(K \mid G)$ denote the set of characteristic vectors for $G^{\prime}(v)$ whose restriction to $G$ agrees with the restriction of $K$, we claim that there is a unique vector in $\mathcal{E}(K \mid G)$ which maximizes $\mathcal{F} \circ \phi$ (so it is $K$ ), and that vector satisfies $\langle K, e\rangle=-1$. To see this, observe that if $L \in \mathcal{E}(K \mid G)$ satisfies $|\langle L, e\rangle|>1$, then $\mathcal{F}(\phi(L))<\mathcal{F}(\phi(K))$. This follows immediately by the adjunction relation: if $L$ satisfies this hypothesis, then by adding or subtracting $2 \mathrm{PD}(e)$ (depending on the sign of $\langle L, e\rangle$ ), we could find a new vector $L^{\prime}$ with $U^{m} \cdot \phi\left(L^{\prime}\right)=\phi(L)$ for $m>0$, so $\mathcal{F}(\phi(L))<\mathcal{F}\left(\phi\left(L^{\prime}\right)\right) \leq \mathcal{F}(\phi(K))$. Next, we claim that $\langle K, e\rangle=-1$, for if $\langle K, e\rangle=+1$, then by adding $2 \mathrm{PD}(e)$, we would obtain a new characteristic vector $K^{\prime}$ with $\phi(K)=\phi\left(K^{\prime}\right)$, but with

$$
\left\langle K^{\prime}, v\right\rangle=\langle K, v\rangle+2,
$$

violating the hypothesis that $K \in \mathcal{K}_{2}$. This completes the proof that $K$ is the unique vector in $\mathcal{E}(K \mid G)$ which maximizes $\mathcal{F} \circ \phi$, so it follows immediately that $\mathbb{A}^{+}(\phi)(K \mid G) \neq 0$.

To verify that $\mathbb{B}^{+} \circ \mathbb{A}^{+}=0$, we proceed as follows. It is an easy consequence of the adjunction relation that if $K \in \operatorname{Char}(G-v)$ is any characteristic vector, then for any $i \geq 0$, we have that

$$
\begin{aligned}
\phi(K, p, 2 i+1) & =U^{\frac{i(i+1)}{2}} \cdot \phi(K, p+2 i+2,-1), \\
\phi(K, p,-(2 i+1)) & =U^{\frac{i(i+1)}{2}} \cdot \phi(K, p-2 i,-1),
\end{aligned}
$$

where $(K, i, j) \in \operatorname{Char}\left(G^{\prime}(v)\right)$ is the characteristic vector whose restriction to $G-v$ is $K$, and its values on $v$ and $e$ are $i$ and $j$ respectively.

Thus in the double sum

$$
\left\langle\left(\mathbb{B}^{+} \circ \mathbb{A}^{+}\right)(\phi), K\right\rangle=\sum_{i=-\infty}^{+\infty} \sum_{j=-\infty}^{+\infty} \phi(K, 2 i+m(v), 2 j+1),
$$


the terms cancel in pairs.

Proposition 2.11 Suppose $G$ is a plumbing diagram satisfying Inequality (8) at each vertex, then $T^{+}$induces an isomorphism

$$
T^{+}: H F^{+}(-Y(G)) \stackrel{\cong}{\longrightarrow} \mathbb{H}^{+}(G) .
$$

Proof With a slight abuse of notation, we let

$$
\widehat{\mathbb{H}}(G(v))=\operatorname{Ker} U \subset \mathbb{H}^{+}(G(v))
$$

throughout this proof.

We prove the result by induction on the graph. The basic cases where the graph is empty (so there is only one characteristic vector, the zero vector) is obvious, as is the case where the graph has a single vertex labelled with multiplicity -1 .

We now prove the result by induction on the number of vertices in the graph, and then a sub-induction on $-m(v)$, where $v$ is a leaf in the graph. For the sub-induction, we allow $m(v)=-1$. This case is handled by the inductive hypothesis on the number of vertices, since $-Y(G)$ is equivalent to a plumbing on a graph with fewer vertices and no bad points, and observing that the identification from Proposition 2.5 is natural under $T^{+}$.

For the sub-inductive step on $-m(v)$, consider the following analogue of Diagram (12) (which also commutes, according to Lemma 2.10):

$$
\begin{array}{ccccc}
0 \longrightarrow & \widehat{H F}\left(-Y\left(G^{\prime}(v)\right)\right) & \widehat{A} & \widehat{H F}(-Y(G)) \stackrel{\widehat{B}}{\longrightarrow} \widehat{H F}(-Y(G-v)) \longrightarrow 0, \\
& \widehat{T}_{G^{\prime}(v)} \downarrow & \widehat{T}_{G} \downarrow & & \widehat{T}_{G-v} \downarrow \\
\left.\widehat{\mathbb{H}}\left(G^{\prime}(v)\right)\right) & \stackrel{\widehat{\mathbb{A}}}{\longrightarrow} \widehat{\mathbb{H}}(G) \quad \stackrel{\widehat{\mathbb{B}}}{\longrightarrow} \quad \widehat{\mathbb{H}}(G-v) .
\end{array}
$$

Here, the top row is exact according to Proposition 2.8. It follows by the inductive hypotheses, that both $\widehat{T}_{G^{\prime}(v)}$ and $\widehat{T}_{G-v}$ are isomorphisms. For $\widehat{T}_{G-v}$, this is obvious, while for $\widehat{T}_{G^{\prime}(v)}$ we use Proposition 2.5. It follows immediately that $\widehat{\mathbb{B}}$ is surjective. Moreover, since $\widehat{\mathbb{B}} \circ \widehat{\mathbb{A}}=0$, and the rank of $\widehat{\mathbb{H}}(G)$ is the sum of the ranks of $\widehat{\mathbb{H}}\left(G^{\prime}(v)\right)$ and $\widehat{\mathbb{H}}(G-v)$ (according to Lemma 2.7), it follows from an easy count of ranks that the kernel of $\widehat{\mathbb{B}}$ is the image of $\widehat{\mathbb{A}}$. Now, by the five-lemma, it follows that $\widehat{T}_{G}$ is an isomorphism.

We now consider Diagram (12), where $v$ is a leaf. Observe that $A^{+}$is injective while $B^{+}$is surjective according to Proposition 2.8. Again, by induction we have that $T_{G^{\prime}(v)}^{+}$and $T_{G-v}^{+}$are isomorphisms, so that $\mathbb{A}^{+}$is injective and $\mathbb{B}^{+}$ 
is surjective. Now, exactness in the middle follows easily from the fact that it holds on the level of $\widehat{H F}$, together with the fact that $\mathbb{B}^{+} \circ \mathbb{A}^{+}=0$. As before, we can now use the five-lemma to establish the desired isomorphism.

Proposition 2.12 Suppose $G$ is a negative-definite plumbing diagram with no bad points, then $T^{+}$induces an isomorphism

$$
T^{+}: H F^{+}(-Y(G)) \stackrel{\cong}{\longrightarrow} \mathbb{H}^{+}(G) .
$$

Proof This is proved by induction on the number of vertices $G$ with $d(v)=$ $-m(v)$. The case where there is no vertex with $d(v)=-m(v)$, is Proposition 2.11. For the inductive step, we consider Diagram (12) again (together with Proposition 2.8), where $v \in G$ is a vertex (not necessarily a leaf) with $d(v)=-m(v)-1$. This time induction tells us that $T_{G-v}^{+}$and $T_{G}^{+}$are isomorphisms. This, together with Lemma 2.10, is sufficient (after a straightforward diagram chase) to allow us to conclude that $T_{G^{\prime}(v)}^{+}$is an isomorphism.

Proof of Theorem 2.1 This follows in from Proposition 2.12 in a manner analogous to how that proposition follows from Proposition 2.11. Again, we consider Diagram (12), now choosing $v$ to be the bad vertex. This time, induction and Proposition 2.12 tells us that $T_{G-v}^{+}$and $T_{G}^{+}$are isomorphisms. Again, we conclude (with the help of Lemma 2.10) that $T_{G^{\prime}(v)}^{+}$is an isomorphism.

Proof of Theorem 2.2 We proceed as in the above proof. Let $v$ be one of the bad vertices in $G$. We would like to prove the result by descending induction on $-m(v)$. In this case, however, Proposition 2.8 is no longer available to us since $G-v$ has a bad vertex; but it is the case that $H F_{\text {odd }}^{+}(-Y(G-v))=0$, in view of Theorem 2.1 (or, more precisely, Corollary 1.4). Thus, we have the diagram:

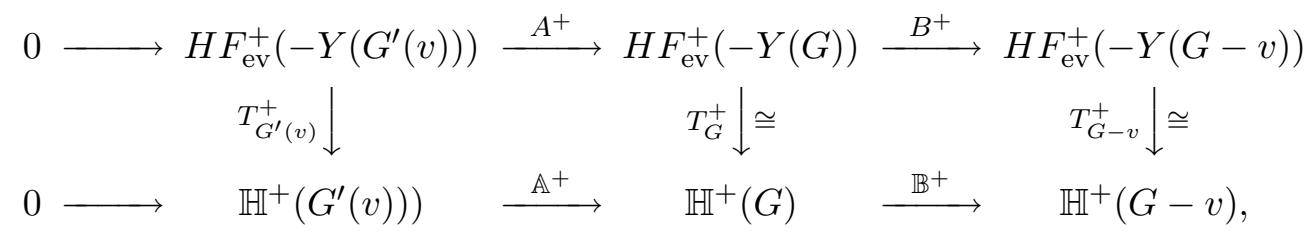

where the maps are indicated as isomorphisms when it follows from induction. This diagram forces $T_{G^{\prime}(v)}^{+}$to be an isomorphism, as well. 
Proof of Corollary 1.5 Fix a $\operatorname{Spin}^{c}$ structure $\mathfrak{t}$ over $Y$, and let $K_{0}$ be a characteristic vector in $\operatorname{Char}_{\mathfrak{t}}(G)$ for which $K^{2}$ is maximal. We define a sequence of elements $\Phi_{N} \in \mathbb{H}^{+}(G, \mathfrak{t})$ by

$$
\Phi_{N}(K)=U^{\left(\frac{K_{0}^{2}-K^{2}}{8}\right)-N} \in \mathcal{T}_{0}^{+} .
$$

As usual, if the exponent of $U$ here is positive, the corresponding element of $\mathcal{T}_{0}^{+}$is zero. so, in particular,

$$
\Phi_{0}(K)= \begin{cases}1 & \text { if } K^{2} \text { is maximal in } \operatorname{Char}_{\mathfrak{t}}(G) \\ 0, & \text { otherwise }\end{cases}
$$

so

$$
\operatorname{deg}\left(\Phi_{0}\right)=-\left(\frac{K_{0}^{2}+|G|}{4}\right) .
$$

Clearly, $U \cdot \Phi_{N+1}=\Phi_{N}$, and $U \cdot \Phi_{0}=0$. Thus, by Theorem 2.1 (and Theorem 2.2, in the case where there are two bad points), it follows that $\operatorname{deg}\left(\Phi_{0}\right)=d(-Y(G), \mathfrak{t})$. Since $d(-Y(G), \mathfrak{t})=-d(Y(G), \mathfrak{t})$, the corollary follows.

\section{$2.1 \quad$ Theorem 1.2 over $\mathbb{Z}$}

Strictly speaking, when working over $\mathbb{Z}$, the map associated to a cobordism as defined in [11] does not have a canonical sign. Thus, it might appear that $T^{+}$ is defined only as a map

$$
T^{+}: H F^{+}(-Y(G), \mathfrak{t}) \longrightarrow \operatorname{Hom}\left(\operatorname{Char}_{\mathfrak{t}}(G), \mathcal{T}_{0}^{+} / \pm 1\right) .
$$

In fact, we can actually specify a map

$$
T^{+}: H F^{+}(-Y(G), \mathfrak{t}) \longrightarrow \operatorname{Hom}\left(\operatorname{Char}_{\mathfrak{t}}(G), \mathcal{T}_{0}^{+}\right),
$$

which is well-defined up to an overall \pm 1 sign, which we pin down with some additional data. Specifically, fix a single $\operatorname{Spin}^{c}$ structure $\mathfrak{s} \in \operatorname{Spin}^{c}(W(G))$ whose restriction to $-Y(G)$ is $\mathfrak{t}$. Since $W(G)$ is a negative-definite cobordism between rational homology spheres, the induced map

$$
F_{W, \mathfrak{s}}^{\infty}: H F^{\infty}(-Y(G), \mathfrak{t}) \longrightarrow H F^{\infty}\left(S^{3}\right)
$$

is an isomorphism (cf. Proposition 9.4 of [15]), and hence determined up to an overall \pm 1 . Now, for each other $\operatorname{Spin}^{c}$ structure $\mathfrak{s}^{\prime}$, we choose orientation conventions so that the induced isomorphism $F_{W, \mathfrak{s}^{\prime}}^{\infty}$ agrees with $U^{m} \cdot F_{W, \mathfrak{s}}^{\infty}$ (where here, of course, $\left.m=\frac{c_{1}(\mathfrak{s})^{2}-c_{1}\left(\mathfrak{s}^{\prime}\right)}{8}\right)$. This fixes signs for all the maps $F_{W(G), \mathfrak{s}^{\prime}}^{+}$. 
With this sign in place, we see that $T^{+}$induces a map to $\mathbb{H}^{+}(G, \mathfrak{t})$ with $\mathbb{Z}$ coefficients (i.e. Proposition 2.4 works over $\mathbb{Z}$ ), which is uniquely determined up to one overall sign.

Before defining $\mathbb{A}^{+}$and $\mathbb{B}^{+}$, we pause for a discussion of their geometric counterparts $A^{+}$and $B^{+}$appearing in the long exact sequence for $\mathrm{HF}^{+}$.

Recall that there was considerable leeway in the orientation conventions used in defining the maps in the surgery long exact sequence for $\mathrm{HF}^{+}$, see [12]. Indeed, the maps $A^{+}$and $B^{+}$are defined as sums of maps induced by cobordisms, and any orientation convention was allowed provided that the composite map (on the chain level) is chain homotopic to 0. More concretely, this can be stated (using notation from Lemma 2.10) as follows. Let $W_{1}$ be the cobordism from $-Y\left(G^{\prime}(v)\right)$ to $-Y(G)$ and $W_{2}$ be the cobordism from $-Y(G)$ to $-Y(G-v)$, and observe that the composite cobordism can be identified as a blow-up $W_{1} \cup$ $W_{2}=W_{3} \# \overline{\mathbb{C P}}^{2}$ (with exceptional sphere $e$ ), then we can let $A^{+}$and $B^{+}$be the maps on homology induced by chain maps:

$$
a^{+}=\sum_{\mathfrak{s}_{1} \in \operatorname{Spin}^{c}\left(W_{1}\right)} \alpha\left(\mathfrak{s}_{1}\right) \cdot f_{W_{1}, \mathfrak{s}_{1}}^{+} \text {and } \quad b^{+}=\sum_{\mathfrak{s}_{2} \in \operatorname{Spin}^{c}\left(W_{2}\right)} \beta\left(\mathfrak{s}_{2}\right) \cdot f_{W_{2}, \mathfrak{s}_{2}}^{+},
$$

where $f^{+}$denotes the chain map induced by the cobordism (with the canonical orientation convention, since both are negative-definite cobordisms in our case), and

$$
\alpha: \operatorname{Spin}^{c}\left(W_{1}\right) \longrightarrow\{ \pm 1\}, \quad \beta: \operatorname{Spin}^{c}\left(W_{2}\right) \longrightarrow\{ \pm 1\}
$$

are maps satisfying the constraint that if $\mathfrak{s}, \mathfrak{s}^{\prime} \in \operatorname{Spin}^{c}\left(W_{1} \# W_{2}\right)$ are any two $\operatorname{Spin}^{c}$ structures which agree over $W_{3}$, and

$$
\left\langle c_{1}(\mathfrak{s}), e\right\rangle=-\left\langle c_{1}\left(\mathfrak{s}^{\prime}\right), e\right\rangle,
$$

then

$$
\alpha\left(\left.\mathfrak{s}\right|_{W_{1}}\right) \cdot \beta\left(\left.\mathfrak{s}\right|_{W_{2}}\right)=-\alpha\left(\left.\mathfrak{s}^{\prime}\right|_{W_{1}}\right) \cdot \beta\left(\left.\mathfrak{s}^{\prime}\right|_{W_{2}}\right)
$$

For example, we can choose $\beta \equiv 1$, and $\alpha$ as follows. Let $\mathrm{PD}[e] \in W_{1} \# W_{2}$, and let $\epsilon=\left.\mathrm{PD}[E]\right|_{W_{1}}$. For each $\mathbb{Z} \epsilon$-orbit in $\operatorname{Spin}^{c}\left(W_{1}\right)$, fix an initial $\operatorname{Spin}^{c}$ structure $\mathfrak{s}_{0}$ over $W_{1}$, and let $\alpha\left(\mathfrak{s}_{0}\right)=1$. Then, if $\mathfrak{s}-\mathfrak{s}_{0}=m \epsilon$, we let $\alpha(K)=(-1)^{m}$.

We now define the maps fitting into the short exact sequence:

$$
\mathbb{A}^{+}: \mathbb{H}^{+}\left(G^{\prime}(v)\right) \longrightarrow \mathbb{H}^{+}(G), \text { and } \mathbb{B}^{+}: \mathbb{H}^{+}(G) \longrightarrow \mathbb{H}^{+}(G-v) .
$$


These maps are defined by the formulas:

$$
\begin{aligned}
\left\langle\mathbb{A}^{+}(\phi),(K, p)\right\rangle & =\sum_{j=-\infty}^{+\infty} \alpha(K, p, 2 j+1) \phi(K, p, 2 j+1), \\
\left\langle\mathbb{B}^{+}(\phi), K\right\rangle & =\sum_{i=-\infty}^{+\infty} \beta(K, 2 i+m(v)) \phi(K, 2 i+m(v)),
\end{aligned}
$$

where here $\alpha(K, p, 2 j+1)$ denotes $\alpha$ applied to the restriction to $W_{1}$ of the Spin $^{c}$ structure over $W\left(G^{\prime}(v)\right)$ whose first Chern class is $(K, p, 2 j+1)$, with the similar shorthand for $\beta$.

With these sign conventions in place, we claim that the analogue of Lemma 2.10 now holds. (Where the statement about commutative squares is weakened to squares which commute, up to sign.) Indeed, all the proofs from the last subsection readily adapt now to prove both Theorems 2.1 and 2.2 over $\mathbb{Z}$.

\section{Calculations}

\subsection{An algorithm for determining the rank of $\operatorname{Ker} U$}

The group $\mathbb{H}^{+}(G)$ can be determined from the combinatorics of the plumbing diagram. In fact, Lemma 2.3 gives us a finite model for $\mathbb{H}^{+}(G)$ (at least, the subset of $\operatorname{Ker} U^{n+1}$, for arbitrary $n$ ). We give here a more practical algorithm for calculating $\operatorname{Ker} U$ or, more precisely, its dual space.

Fix a characteristic vector $K$ satisfying inequality

$$
m(v)+2 \leq\langle K, v\rangle \leq-m(v) .
$$

Now, successively apply the following algorithm to find a path of vectors $(K=$ $\left.K_{0}, K_{1}, \ldots, K_{n}\right)$ so that the $K_{i}$ for $i<n$ satisfy the bounds

$$
\left|\left\langle K_{i}, v\right\rangle\right| \leq-m(v)
$$

for all vertices $v$. Given $K_{i}$, choose any vertex $v_{i+1}$ with

$$
\left\langle K_{i}, v_{i+1}\right\rangle=-m\left(v_{i+1}\right) \text {, then let } K_{i+1}=K_{i}+2 \operatorname{PD}\left[v_{i+1}\right] .
$$

This algorithm can terminate in one of two ways: either

- the final vector $L=K_{n}$ satisfies the inequality,

$$
m(v) \leq\langle L, v\rangle \leq-m(v)-2
$$

at each vertex $v$ or 
- there is some vertex $v$ for which

$$
\left\langle K_{n}, v\right\rangle>-m(v) .
$$

To calculate the rank of $\operatorname{Ker} U$ in the examples in this paper, we use the following claim: the equivalence classes in $\mathbb{K}^{+}(G)$ which have no representative of the form $U^{m} \otimes K^{\prime}$ with $m>0$ are in one-to-one correspondence with initial vectors $K$ satisfying Inequality (13) for which the algorithm above terminates in a characteristic vector $L$ satisfying Inequality (16). The purpose of Proposition 3.2 is to establish this claim.

Definition 3.1 We call a sequence of characteristic vectors $\left(K=K_{0}, \ldots, K_{n}\right.$ $=L$ ) obtained from the above algorithm a full path; i.e. $K=K_{0}$ satisfies Inequality (13), $K_{i+1}$ is obtained from $K_{i}$ by Equation (15), and the final vector $L=K_{n}$ satisfies either Inequality (16) or (17).

Proposition 3.2 Fix an equivalence class in $\mathbb{K}^{+}(G)$ which contains no representatives of the form $U^{m} \otimes K^{\prime}$ for $m>0$. Each such equivalence class has a unique representative $K$ satisfying Equation (13). Indeed, a characteristic vector $K$ satisfying these bounds is inequivalent to an element of the form $U^{m} \otimes K^{\prime}$ (with $m>0$ ) if and only if we can find a full path

$$
\left(K=K_{0}, K_{1}, \ldots, K_{n}=L\right)
$$

terminating with a characteristic vector $L=K_{n}$ which satisfies Inequality (16) for each vertex $v$.

Proof Let $M$ be a characteristic vector which is not equivalent to $U^{m} \otimes K^{\prime}$ for $m>0$. We find a full path using the above algorithm. Specifically we let $L_{0}=M$, and then for each $j \geq 0$, and extend $L_{0}$ to a sequence $L_{0}, \ldots, L_{n_{+}}$ by letting $v_{j+1}$ be a vertex for which

$$
\left\langle L_{j}, v_{j+1}\right\rangle=-m\left(v_{j+1}\right),
$$

and then letting $L_{j+1}=L_{j}+2 \mathrm{PD}\left[v_{j+1}\right]$. Clearly, in this sequence, each element satisfies

$$
m(v) \leq\left\langle L_{j}, v\right\rangle \leq-m(v)
$$

(for otherwise, $L_{j}$ would be equivalent to an element of $\mathbb{Z}^{>0} \times \operatorname{Char}(G)$ ). The sequence is finite (since the elements of the sequence are all distinct), so it must terminate with $L_{n_{+}}$satisfying Inequality (16). In the same way, we can extend back from $L_{0}$ to obtain a sequence $\left(L_{0}, L_{-1}, \ldots, L_{n_{-}}\right)$by the rule that if there is a vertex $v$ for which $\left\langle L_{j}, v\right\rangle=m(v)$, then

$$
L_{j-1}=L_{j}-2 \mathrm{PD}[v] .
$$


This sequence must terminate with $L_{n_{-}}$satisfying Inequality (13). Thus,

$$
\left(L_{n_{-}}, L_{n_{-}+1}, \ldots, L_{0}=M, L_{1}, \ldots, L_{n_{+}}\right)
$$

is a full path in the sense of Definition 3.1. In particular, $L_{n_{-}}$is the representative $K$.

We argue that if $M$ is a vector in a full path $\left(K=K_{0}, \ldots, K_{n}=L\right)$, so that $L$ satisfies Inequality (16), then $L$ is uniquely determined by $M$ (i.e. independent of the particular sequence). In fact, if $\left\{v_{1}, \ldots, v_{\ell}\right\}$ are vertices with $\left\langle M, v_{i}\right\rangle=$ $-m\left(v_{i}\right)$, then $L$ must be obtained from $M$ by adding $2 \mathrm{PD}\left[v_{1}\right]+\cdots+2 \mathrm{PD}\left[v_{\ell}\right]$ (so that we can achieve $\left\langle L, v_{i}\right\rangle<-m\left(v_{i}\right)$ ), and then adding some additional vertices. Thus,

$$
M+2 \mathrm{PD}\left[v_{1}\right]+\cdots+2 \mathrm{PD}\left[v_{\ell}\right]
$$

lies on a full path with the same endpoint $L$. By induction on the minimal distance of $M$ to its endpoint on a full path, we have the uniqueness of the final point $L$.

Next, we argue that if $M$ and $M^{\prime}$ are two characteristic vectors which are equivalent to one another, and $M \nsim U^{m} \otimes K^{\prime}$ for $m>0$, then the endpoint of any full path through $M$ agrees with the endpoint of a full path through $M^{\prime}$. This is clear if $M^{\prime}=M \pm 2 \mathrm{PD}[v]$ : we can find a full path which passes through both $M$ and $M^{\prime}$. More generally, if $M \sim M^{\prime}$, we can get from $M$ to $M^{\prime}$ by a finite number of additions or subtractions of $2 \mathrm{PD}[v]$ for vertices so as to leave the square unchanged (i.e. $M_{i+1}$ is obtained from $M_{i}$ by $M_{i+1}=M_{i}-$ $2 \epsilon_{i+1} \mathrm{PD}\left[v_{i+1}\right]$ where $v_{i+1}$ is a vertex which satisfies $\left\langle M_{i}, v_{i+1}\right\rangle=\epsilon_{i+1} m\left(v_{i+1}\right)$ for $\epsilon_{i+1}= \pm 1$ ). The assertion then follows by an easy induction on the number of such operations.

Turning this around, we also see that the initial point of a full path is uniquely determined by the equivalence class of the characteristic vectors lying in it. This gives the uniqueness statement claimed in the proposition.

Finally, we argue that if $K$ is a vector satisfying Inequality (13), but $K \sim$ $U^{m} \otimes K^{\prime}$ for $m>0$, then there is no full path connecting $K$ to another characteristic vector $L$ satisfying Inequality (16). To see this suppose that $K \sim U^{m} \otimes K^{\prime}$, we can find some sequence

$$
M_{0}=K, M_{1}, \ldots, M_{\ell}=M
$$

and signs $\epsilon_{i} \in\{ \pm 1\}$ with

$$
M_{i+1}=M_{i}-2 \epsilon_{i+1} \mathrm{PD}\left[u_{i+1}\right]
$$


and

$$
\left\langle M_{i},\left[u_{i+1}\right]\right\rangle=\epsilon_{i+1} m\left(u_{i+1}\right) .
$$

where each $M_{i}$ satisfies (for each vertex $v$ )

$$
\left|\left\langle M_{i},[v]\right\rangle\right| \leq-m(v)
$$

but there is some vertex $w$ with $|\langle M,[w]\rangle|>-m(v)$. We claim that this sequence can be shortened so that all the $\epsilon_{i}$ are positive. To see this, observe first that $\epsilon_{1}=+1$. Now, consider the smallest integer $i$ with $\epsilon_{i+1}=-1$, so that $M_{i} \cdot u_{i+1}=m\left(u_{i+1}\right)$. It follows easily that $u_{i+1} \in\left\{u_{1}, \ldots, u_{i}\right\}$. If we let $j$ be the last integer in $[1, \ldots, i]$ with $u_{j}=u_{i+1}$, then it is easy to see that for each $k \in[j+1, \ldots, i], u_{k} \cdot u_{i+1}=0$. It follows immediately that we delete the occurance of the $j^{\text {th }}$ and $(i+1)^{\text {st }}$ vertices from $\left(u_{1}, \ldots, u_{n}\right)$ to construct a new, shorter sequence on characteristic vectors satisfying the same properties as the $M_{i}$ (in particular, connecting the same two endpoints), only with one fewer occurance of the sign $\epsilon_{j}=-1$. Proceeding in this manner, we end up with a sequence with all $\epsilon_{j}=+1$.

Next, suppose that there is a full path connecting $K$ as above to $L$. We claim now that, after possibly reordering, $\left(u_{1}, \ldots, u_{\ell}\right)$ is a subsequence of the vertices $\left(v_{1}, \ldots, v_{n}\right)$ belonging to the hypothesized full path connecting $K$ to $L$. It is easy to see then that we can extend the original sequence $\left(K=M_{0}, \ldots, M_{\ell}\right)$ to a sequence $K=M_{0}, \ldots, M_{n}=L$ (using a reordering $\left(w_{1}, \ldots, w_{n}\right)$ of $\left(v_{1}, \ldots, v_{n}\right)$ ) so that

$$
M_{i+1}=M_{i}+2 \mathrm{PD}\left[w_{i+1}\right]
$$

and $\left\langle M_{i},\left[w_{i+1}\right]\right\rangle \geq-m\left(w_{i+1}\right)$. This forces $K \sim L \sim U^{m} \otimes L$ for $m>0$. But it is impossible for $U^{m} \otimes L \sim L$.

\subsection{Examples}

We illustrate the algorithm described above by calculating $\mathrm{HF}^{+}(Y)$ for certain Brieskorn spheres $Y$.

Notational Conventions In describing graded $\mathbb{Z}[U]$-modules, we adopt the following conventions. $\mathcal{T}_{k}^{+}$will denote the graded $\mathbb{Z}[U]$-module which is isomorphic as a relatively graded $\mathbb{Z}[U]$-module to $\mathrm{HF}^{+}\left(S^{3}\right)$, but whose bottom-most non-zero homogeneus element has degree $k$. Also, $\mathbb{Z}_{(k)}$ will denote the $\mathbb{Z}[U]$ module $\mathbb{Z}[U] / U \mathbb{Z}[U]$, graded so that is supported in degree $k$.

For example, with this notation,

$$
H F^{+}\left(S^{2} \times S^{1}\right) \cong \mathcal{T}_{-1 / 2}^{+} \oplus \mathcal{T}_{1 / 2}^{+}
$$


The Poincaré homology sphere Consider the Poincaré homology sphere $Y=\Sigma(2,3,5)$. This can be realized as the boundary of the plumbing of spheres specified by the negative-definite $E_{8}$ Dynkin diagram.

We claim that the techniques of the present paper can be used to verify that

$$
H F^{+}(-\Sigma(2,3,5)) \cong \mathcal{T}_{-2}^{+},
$$

compare Section 8 of [15].

We claim that the only full path connecting vectors $K$ and $L$ as in Proposition 3.2 is the path consisting of the single characteristic vector $K=L=0$.

Specifically, we consider the 256 possible initial characteristic vectors $K$ as in Proposition 3.2, i.e.

$$
\left\langle K, v_{i}\right\rangle \in\{0,2\} .
$$

It is easy to see that if $\left\langle K, v_{i}\right\rangle=2$ for at least two vertices, then the algorithm given above terminates with a characteristic vector $L$ satisfying Inequality (17): i.e. $K \sim U \otimes K^{\prime}$.

It remains then to rule out eight remaining cases where there is only one vertex on which $K$ does not vanish. Ordering the vertices in the $E_{8}$ diagram so that $v_{1}$ is the central node (with degree three), and $\left(v_{1}, v_{2}\right),\left(v_{1}, v_{3}, v_{4}\right)$, and $\left(v_{1}, v_{5}, v_{6}, v_{7}, v_{8}\right)$ are three connected segments, we write characteristic vectors as tuples

$$
\left(\left\langle K, v_{1}\right\rangle, \ldots\left\langle K, v_{8}\right\rangle\right) .
$$

We include here one of these eight cases - exhibiting a full path from $K_{0}=$ $(0,0,0,0,0,0,0,2)$ to a vector $K_{n}$ with $\left\langle K_{n}, v\right\rangle=4$ for some vertex $v$ - leaving the remaining seven cases to the reader.

$$
\begin{array}{ll}
\{(2,0,0,0,0,0,0,0), & (-2,2,2,0,2,0,0,0), \quad(0,-2,2,0,2,0,0,0), \\
(2,-2,-2,2,2,0,0,0), & (-2,0,0,2,4,0,0,0)\}
\end{array}
$$

The Brieskorn sphere $\Sigma(2,3,7)$ We give here another calculation showing that

$$
H F^{+}(-\Sigma(2,3,7)) \cong \mathcal{T}_{0}^{+} \oplus \mathbb{Z}_{(0)}
$$

(compare [15]).

This homology sphere is realized as the boundary of a plumbing diagram with a central node $v_{1}$ of square -1 , and three more spheres $v_{2}, v_{3}$, and $v_{4}$ of squares $-2,-3$, and -7 respectively. The vectors $(1,0,-1,-3),(1,0,-1,-5)$ are the only two vectors satisfying Inequality (13) which begin a full path ending in a 
characteristic vector as in Inequality (16). For convenience, we include the full path starting at $(1,0,-1,-5)$ :

$$
\begin{array}{llll}
\{(1,0,-1,-5), & (-1,2,1,-3), & (1,-2,1,-3), & (-1,0,3,-1), \\
(1,0,-3,-1), & (-1,2,-1,1), & (1,-2,-1,1), & (-1,0,1,3)\} .
\end{array}
$$

(The other full path is obtained by multiplying all above vectors by -1 and reversing the order.) Now, we claim that $U \otimes(1,0,-1,-3) \sim U \otimes(1,0,-1,-5)$. In fact, it is straightforward to verify that:

$$
U \otimes(-1,0,1,5) \sim(1,0,1,-9) \sim(-1,0,5,-5) \sim U \otimes(1,0,-1,-5) .
$$

Here, we have broken the equivalence up so that when we write $K \sim K^{\prime}$, we mean that $K^{\prime}$ is obtained from $K$ by applying the algorithm for constructing a full path.

It is interesting to note that it follows from the above calculations that the conjugation action, which in general gives a an involution on $\mathrm{HF}^{+}(Y)$, in the present case permutes the two zero-dimensional generators. Observe also that the renormalized length $\left(\frac{K^{2}+|G|}{4}\right)$ of both vectors is 0 .

The Brieskorn sphere $\Sigma(3,5,7)$ We claim that

$$
H F^{+}(-\Sigma(3,5,7)) \cong \mathcal{T}_{-2}^{+} \oplus \mathbb{Z}_{(-2)} \oplus \mathbb{Z}_{(0)} \oplus \mathbb{Z}_{(0)} .
$$

We can realize $\Sigma(3,5,7)$ as the boundary of a negative-definite plumbing of spheres, as in Figure 1. Unlabeled vertices all have multiplicity -2 . We order the vertices so that the central node comes first, the -3 -sphere second, then the four vertices on the next chain (ordered so that the length to the central node is increasing) and finally, the six vertices on the final chain (ordered in the same manner).

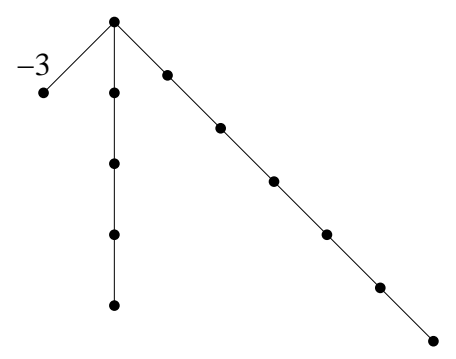

Figure 1: Plumbing description of $\Sigma(3,5,7)$ Here, the unlabeled vertices have multiplicity -2 . 
We claim that there are exactly four characteristic vectors satisfying Inequality (13) which can be completed to full paths terminating in characteristic vectors satisfying Inequality (16), and these are the vectors:

$$
\begin{aligned}
& K_{1}=(0,-1,0,0,0,0,0,0,0,0,0,0) \\
& K_{2}=(0,1,0,0,0,0,0,0,0,0,0,0) \\
& K_{3}=(0,1,0,0,0,0,0,0,0,0,0,-2) \\
& K_{4}=(0,1,0,0,0,-2,0,0,0,0,0,0)
\end{aligned}
$$

It is straightforward to verify that

$$
\begin{aligned}
U \otimes K_{3} & \sim(2,-5,0,0,0,0,0,0,0,0,0,-2) \\
& \sim(0,-1,0,0,0,0,0,0,-2,4,0,-2) \\
& \sim U \otimes(0,-1,0,0,0,0,0,0,0,0,2,-2) \\
& \sim U \otimes(0,-1,0,0,0,0,0,0,0,0,0,2) \\
& \sim U \otimes K_{4} .
\end{aligned}
$$

Here, as before, we break the equivalence up into simpler steps, writing $K \sim K^{\prime}$ if $K^{\prime}$ is obtained from $K$ by applying the algorithm for constructing a full path.

Also we have that

$$
\begin{aligned}
U \otimes K_{1} & \sim(-2,5,0,0,0,0,0,0,0,0,0,0) \\
& \sim(0,1,0,0,0,0,0,0,2,-4,0,0) \\
& \sim U \otimes(0,1,0,0,0,0,0,0,0,0,-2,0) \\
& \sim U \otimes(0,-1,0,0,0,0,0,0,0,2,0,-4) \\
& \sim U^{2} \otimes(0,-1,0,0,0,0,0,0,0,2,-2,0) \\
& \sim U^{2} \otimes K_{4} .
\end{aligned}
$$

A similar calculation shows that

$$
U \otimes K_{2} \sim U^{2} \otimes K_{3} \sim U^{2} \otimes K_{4} .
$$

The result then follows.

\section{The Floer homology of $\Sigma_{2} \times S^{1}$}

As an application of the calculations for plumbing diagrams, we calculate $\mathrm{HF}^{+}$ of the product of a genus two surface with the circle. Along the way, we also calculate $\mathrm{HF}^{+}$for certain other genus two fiber-bundles over the circle. 
Let $T_{2} \subset S^{3}$ denote the connected sum of two copies of the right-handed trefoil $T$. Now, if $Y_{n}=S_{n}^{3}\left(T_{2}\right)$ denote the three-manifold obtained by $+n$-surgery on $S^{3}$ along $T_{2}$, then this manifold can be realized by plumbing along the tree pictured in Figure 2. Note that this graph has at least two bad vertices. However, for $n=+12$, after a handleslide followed by a handle cancellation, we obtain an alternate description of $Y_{12}$ as the Seifert fibered space whose plumbing diagram is pictured in Figure 3.

Note that the realization of $Y_{n}$ as surgery on a knot gives a correspondence

$$
Q: \mathbb{Z} / n \mathbb{Z} \longrightarrow \operatorname{Spin}^{c}\left(Y_{n}\right) \text {. }
$$

We adopt here the conventions for the integral surgery long exact sequence, $\mathrm{cf}$. Theorem 9.19 of [12]. According to these conventions (cf. Lemma 7.10 of [15]), if $W$ denotes the cobordism from $S^{3}$ to $Y_{n}$ obtained by attaching the twohandle, and $[F] \in H_{2}(W ; \mathbb{Z})$ is a generator, then $Q(0)$ is the $\operatorname{Spin}^{c}$ structure over $Y_{n}$ which has an extension $\mathfrak{s}$ over $W$ with

$$
\left\langle c_{1}(\mathfrak{s}),[F]\right\rangle=n .
$$

Lemma 4.1 Let $Q(0)$ be the $\operatorname{Spin}^{c}$ structure over $Y_{12}$ as above on the zerosurgery on the double-trefoil. Then,

$$
H F^{+}\left(-Y_{12}, Q(0)\right) \cong \mathbb{Z}_{(-3 / 4)} \oplus \mathcal{T}_{-3 / 4}^{+} .
$$

Equivalently,

$$
H F^{+}\left(Y_{12}, Q(0)\right) \cong \mathbb{Z}_{(-1 / 4)} \oplus \mathcal{T}_{3 / 4}^{+}
$$

Proof Consider the plumbing diagram $G$ for $Y_{12}$ in Figure 3. We argue that $\operatorname{Ker} U \subset \mathbb{H}^{+}(G, Q(0))$ is two-dimensional.

We order the spheres $S_{1}, S_{2}, S_{3}, S_{4}, S_{5}$, so that $S_{1}$ is the central sphere, and $S_{2}$ and $S_{3}$ are the other two two-spheres with square -2 . We identify characteristic vectors as quintuples, according to the values on $S_{1}, \ldots, S_{5}$.

We use Lemma 2.3 and Proposition 3.2 to calculate $\operatorname{Ker} U$. Of the seventy-two characteristic vectors satisfying Inequality (13) the following six are the only ones which represent the given $\operatorname{Spin}^{c}$ structure:

$$
\begin{array}{lll}
(0,2,2,3,3), & (0,0,0,3,3), & (0,2,2,1,1), \\
(0,0,0,1,1), & (0,2,2,-1,-1), & (0,0,0,-1,-1) .
\end{array}
$$

Indeed, we claim that of these six characteristic vectors, $K=(0,0,0,1,1)$ and $-K$ are the only two which can be connected to characteristic vectors satisfying Inequality (16). For example,

$$
(0,2,2,3,3) \sim(4,2,2,-3,-3) \sim U \otimes(0,4,4,-1,-1) .
$$


Moreover, since

$$
\frac{K^{2}+5}{4}=\frac{3}{4},
$$

it follows immediately that the kernel of $U$ inside $\mathbb{H}^{+}(G)$ has rank two, and it is supported in degree $-3 / 4$.

Next, we claim that the kernel of $U^{2}$ has rank three. This follows from the fact that

$$
U \otimes K \sim U \otimes-K
$$

more specifically:

$(4,-2,-2,-3,-3) \sim(2,-2,-2,-3,3) \sim(-2,0,0,-1,5) \sim U \otimes(0,0,0,-1,-1)$,

while

$$
(4,-2,-2,-3,-3) \sim(-2,2,2,-3,3) \sim(2,0,0,-5,1) \sim U \otimes(0,0,0,1,1) .
$$

The restatement for $Y_{12}$ (rather than $-Y_{12}$ ) follows from the general properties of the invariant under orientation reversal.

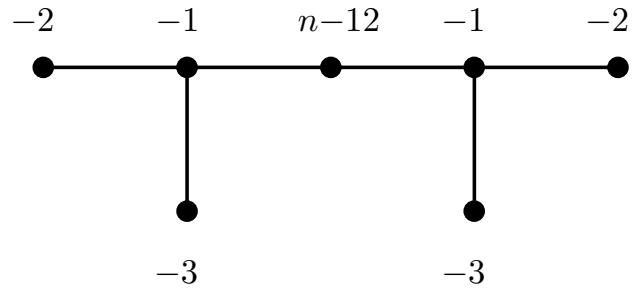

Figure 2: Plumbing description of a connected sum of two trefoils Here, for an arbitrary integer $n$, we have a description of $Y_{n}=S_{n}^{3}\left(T_{2}\right)$ as a plumbing diagram.

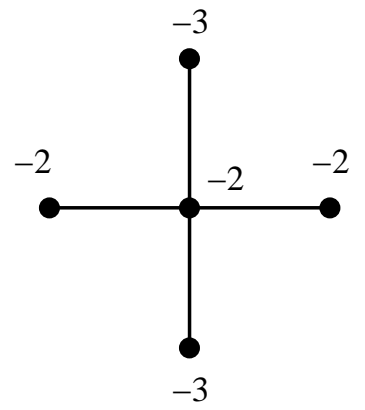

Figure 3: Plumbing description for $Y_{12}$ 
Proposition 4.2 Let $Y_{0}$ denote zero-surgery on the connected sum $T_{2}$ of two copies of the right-handed trefoil. Then, under the identification $\operatorname{Spin}^{c}\left(Y_{0}\right) \cong 2 \mathbb{Z}$ (using the first Chern class and then a trivialization $H^{2}\left(Y_{0} ; \mathbb{Z}\right) \cong \mathbb{Z}$ ), we have that

$$
\begin{aligned}
H F^{+}\left(Y_{0}, \pm 2\right) & \cong \mathbb{Z} \\
H F^{+}\left(Y_{0}, m\right) & =0
\end{aligned}
$$

for $|m|>2$. Moreover, as a $\mathbb{Z}[U]$ module, we have that

$$
H F^{+}\left(Y_{0}, 0\right) \cong \mathcal{T}_{-1 / 2}^{+} \oplus \mathcal{T}_{-3 / 2}^{+} \oplus \mathbb{Z}_{(-5 / 2)}
$$

(the subscript on the last factor here denotes the absolute grading of the $\mathbb{Z}$ summand).

Proof The fact that $\mathrm{HF}^{+}\left(Y_{0}, m\right)=0$ for $|m|>2$ follows from the adjunction inequality for $\mathrm{HF}^{+}$(cf. Theorem 7.1 of [12]). The fact that $H F^{+}\left(Y_{0}, \pm 2\right) \cong \mathbb{Z}$ follows from the fact that $Y_{0}$ is a genus two fibered knot (cf. Theorem 5.2 of [14]). (An alternative verification of these facts could be given by a more extensive calculation of $Y_{12}$, in the spirit of Lemma 4.1.)

We now use the graded version of the integral surgeries long exact sequence (cf. Section 7 of [15]) to determine $H^{+}\left(Y_{0}, 0\right)$. Recall that that sequence gives:

$\cdots \longrightarrow H^{+}\left(S^{3}\right) \stackrel{F_{1}}{\longrightarrow} H F^{+}\left(Y_{0}, 0\right) \stackrel{F_{2}}{\longrightarrow} H F^{+}\left(Y_{12}, Q(0)\right) \stackrel{F_{3}}{\longrightarrow} \cdots$

(in general, the term involving $Y_{0}$ reads

$$
\bigoplus_{k \in \mathbb{Z}} H F^{+}\left(Y_{0}, 12 k\right)
$$

but it follows from what we have already seen that $H F^{+}\left(Y_{0}, 0\right)$ is the only nontrivial summand here). The map $F_{3}$ annihilates $H F^{\infty}\left(Y_{12}, Q(0)\right)$, and it can be written as a sum of terms which decrease degree by at least $-11 / 4$. Since $H F_{\text {red }}^{+}\left(Y_{12}, Q(0)\right)$ is supported in degree $-\frac{1}{4}$, it follows immediately that $F_{3}$ is trivial. Bearing in mind that $F_{2}$ is a homogeneous map which shifts degrees by 9/4 (cf. Lemma 7.11 of [15]), the result now follows easily.

Remark 4.3 It is an easy consequence of this calculation that, if $Y_{-1}$ denotes the three-manifold obtained by $(-1)$ surgery on the double-trefoil $T_{2}$, then $H F_{\text {red }}^{+}\left(Y_{-1}\right)$ has generators with both parities; therefore, so does $H F_{\text {red }}^{+}\left(-Y_{-1}\right)$. Note that the plumbing diagram for $Y_{-1}$ in Figure 2 has two bad points (two vertices with degree three and multiplicity -1 ). This underscores the importance of the hypothesis on the graph for Theorem 1.2. 
We now calculate $H F^{+}\left(S^{1} \times \Sigma_{2}\right)$. To do this, we think of $S^{1} \times \Sigma_{2}$ as a surgery on a generalized Borromean rings (compare this with the corresponding calculation of $\mathrm{HF}^{+}\left(T^{3}\right)$ from Section 8 of [15]). Specifically, consider the link pictured in Figure 4. For integers $a, b, c, d$,e, we let $M(a,(b, c)(d, e))$ denote the three-manifold obtained by surgery instructions as labelled in the figure (i.e. $a$ is the coefficient on the long circle). In particular, it is easy to see that $M(0,(1,1)(1,1))$ is zero-surgery on the connected sum of two right-handed trefoils; while $M(0,(0,0)(0,0)) \cong S^{1} \times \Sigma_{2}$.

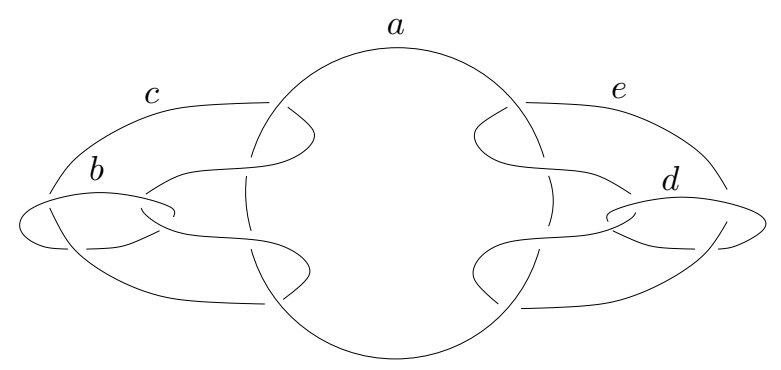

Figure 4: Generalized Borromean rings This link has the property that if the surgery coefficients $a=b=c=d=e=0$, then the three-manifold obtained is $S^{1} \times \Sigma_{2}$.

We calculate $M(0,(0,0),(0,0))$ by successive applications of the long exact sequence. In this calculation, we will make heavy use of what is known about $H F^{\infty}$ (see Section 10 of [12]). Recall that a three-manifold $Y$ is said to have standard $H F^{\infty}$ if for each torsion $\operatorname{Spin}^{c}$ structure $\mathfrak{t}_{0}$,

$$
H F^{\infty}\left(Y, \mathfrak{t}_{0}\right) \cong \mathbb{Z}\left[U, U^{-1}\right] \otimes_{\mathbb{Z}} \Lambda^{*} H^{1}(Y ; \mathbb{Z})
$$

as a $\mathbb{Z}\left[U, U^{-1}\right] \otimes_{\mathbb{Z}} \Lambda^{*} H_{1}(Y ; \mathbb{Z})$-module. In general, we have a spectral sequence whose $E_{2}$ term is $\mathbb{Z}\left[U, U^{-1}\right] \otimes_{\mathbb{Z}} \Lambda^{*} H^{1}(Y ; \mathbb{Z})$ which converges to $H F^{\infty}\left(Y, \mathfrak{t}_{0}\right)$, so this condition is equivalent to the condition that all higher differentials $d_{r}$ for $r \geq 2$ are trivial. Three-manifolds with $b_{1}(Y) \leq 2$ all have standard $H F^{\infty}$. Moreover, if $Y$ has standard $H F^{\infty}$, and $\mathbb{K} \subset Y$ is a framed, null-homologous knot, and $b_{1}\left(Y_{\mathbb{K}}\right)=b_{1}(Y)$, then $Y_{\mathbb{K}}$ also has standard $H F^{\infty}$ (cf. Proposition 9.4 of [15]).

Thus, in our exact sequences, we will find it convenient to work with the threemanifolds $Y=M(a(b, c)(d, e))$ with $a=1$ as much as possible, since all of these three-manifolds have standard $H F^{\infty}$. The cost is that these three-manifolds have two extra generators in $\mathrm{HF}^{+}$. (We will not need to calculate their absolute gradings, however.)

More precisely, we have the following: 
Lemma 4.4 Fix any integers $b, c, d, e \in\{0,1\}$, and let $Z_{0}$ denote the threemanifold $Z_{0}=M(0,(b, c),(d, e))$. We have an identification

$$
\bigoplus_{\left\{\mathfrak{t} \in \operatorname{Spin}^{c}\left(Z_{0}\right) \mid c_{1}(\mathfrak{t}) \neq 0\right\}} H F^{+}\left(Z_{0}, \mathfrak{t}\right) \cong \mathbb{Z}^{2} .
$$

Moreover, if $Z_{1}=M(1,(b, c),(d, e))$, this subgroup injects into $H F^{+}\left(Z_{1}\right)$. Similarly, the corresponding subgroup of $\widehat{H F}\left(Z_{0}\right)$ has rank four, and it, too, injects into $\widehat{H F}\left(Z_{1}\right)$.

Proof The first claim follows from the fact that $Z_{0}$ is a genus two fibration (see [14]). The injectivity claim follows from the long exact sequence connecting $Z_{0}, Z_{1}$, and a third term which is a connected sum of some number of copies of $S^{1} \times S^{2}$.

We will let $V_{(b, c)(d, e)} \subset H F^{+}(M(1(b, c)(d, e)))$ denote the rank two subgroup constructed in Lemma 4.4 , and $\widehat{V}_{(b, c)(d, e)} \subset \widehat{H F}(M(1(b, c)(d, e)))$ be the corresponding rank four subgroup. ${ }^{1}$

Lemma 4.5 We have $\mathbb{Z}[U]$-module identifications:

$$
\begin{aligned}
& H F^{+}(M(1(1,1),(1,1))) \cong \mathcal{T}_{-2}^{+} \oplus \mathbb{Z}_{(-3)} \oplus V_{(1,1),(1,1)} \\
& H F^{+}(M(1(1,0),(1,1))) \cong \mathcal{T}_{-5 / 2}^{+} \oplus \mathcal{T}_{-3 / 2}^{+} \oplus \mathbb{Z}_{(-5 / 2)} \oplus V_{(1,0),(1,1)}
\end{aligned}
$$

Proof Both are relatively straightforward applications of the surgery long exact sequence for $\mathrm{HF}^{+}$, given the calculation from Proposition 4.2.

The surgery exact sequence for the triple

$$
\left(M(\infty(1,1),(1,1)) \cong S^{3}, \quad M(0(1,1)(1,1)), \quad M(1(1,1),(1,1))\right)
$$

reads:

$\cdots \rightarrow \mathcal{T}_{0}^{+} \stackrel{F_{1}}{\longrightarrow} \mathcal{T}_{-1 / 2}^{+} \oplus \mathcal{T}_{-3 / 2}^{+} \oplus \mathbb{Z}_{(-5 / 2)} \oplus W \stackrel{F_{2}}{\longrightarrow} H F^{+}(M(1(1,1),(1,1))) \stackrel{F_{2}}{\longrightarrow} \cdots$

where here $W$ is the rank two module generated by the sum of $\mathrm{HF}^{+}\left(Y_{0}, m\right)$ with $m \neq 0$. Now, we claim that the map $F_{3}$ is trivial. Clearly the map

\footnotetext{
${ }^{1}$ The Abelian groups which we meet now and in the rest of this paper are free $\mathbb{Z}$ modules. To verify this, one can run the exact sequence arguments below for $\mathbb{Z} / p \mathbb{Z}$ where $p$ is an arbitrary prime, and observe that the dimensions of each of the vector spaces in question is independent of the prime $p$, and hence, by the universal coefficients theorem, these groups are free $\mathbb{Z}$-modules. Having said this now, we do not call the readers' attention to it again.
} 
is written as a sum of maps which induce the trivial map on $H F^{\infty}$ (this is necessary in order for $H F^{\infty}(M(0(1,1),(1,1)))$ to have its structure). Thus, $F_{3}$ factors through $H F_{\text {red }}^{+}(M(1(1,1)(1,1)))$. Indeed, it is also trivial on the image $V_{(1,1)(1,1)}$ of $W$ inside $M(1(1,1)(1,1))$ (by exactness). But now, since $F_{2}$ lowers degree by $1 / 2$, this quotient group is isomorphic to a single $\mathbb{Z}$ in dimension -3 . Since the map $F_{3}$ does not increase degree, and $H F^{+}\left(S^{3}\right)$ is supported in non-negative dimension, it follows that $F_{3}$ is trivial. Now, $F_{1}$ is injective, it clearly maps onto the summand $\mathrm{HF}^{+}\left(Y_{0}, 0\right) \subset H F^{+}\left(Y_{0}\right)$. The first isomorphism claimed in the lemma now follows.

The second isomorphism follows from considering the surgery long exact sequence for the triple

$$
(M(1(1, \infty),(1,1)) \cong-\Sigma(2,3,5), \quad M(1(1,0)(1,1)), \quad M(1(1,1),(1,1))) .
$$

Recall that $H^{+}(-\Sigma(2,3,5)) \cong \mathcal{T}_{-2}^{+}$(see [15]). Also, the map in the exact sequence which takes $H F^{+}(M(1(1,0)(1,1)))$ to $H F^{+}(M(1(1,1)(1,1)))$ carries the subgroup $V_{(1,0)(1,1)}$ to $V_{(1,1)(1,1)}$. This latter observation follows from naturality of the maps induced by cobordisms, togther with the observation that the corresponding cobordism connecting $M(0(1,0)(1,1))$ to $M(0(1,1)(1,1))$ induces an isomorphism on the part of $\mathrm{HF}^{+}$supported in $\mathrm{Spin}^{c}$ structures with non-trivial first Chern class. This follows easily from the long exact sequence connecting these $\mathrm{HF}^{+}$of these three-manifolds. Indeed, the point here is that the cobordism from $M(0(1,0)(1,1))$ to $M(0(1,1)(1,1))$ admits a genus two Lefschetz fibration, see [14], especially Lemma 5.4 of that paper.

As before, the map in the long exact sequence taking

$$
H F^{+}(M(1(1,1),(1,1))) \longrightarrow H F^{+}(-\Sigma(2,3,5))
$$

is trivial on the image of $H F^{\infty}$, and it is also trivial on $V_{(1,1),(1,1)}$ (by exactness). The remaining quotient group is a $\mathbb{Z}$ in dimension -3 , and since the map under consideration does not increase degree, and $\mathrm{HF}^{+}(-\Sigma(2,3,5))$ is supported in degrees $\geq-2$, it follows that the map under consideration is trivial.

Remark 4.6 In view of the above calculations, we see that $M(1(1,0)(1,1))$ gives yet another example of an integral homology $S^{2} \times S^{1}$ which is obtained as integral surgery on a two-component link in $S^{3}$, but which is not surgery on any knot (indeed, with the given orientation, this manifold cannot bound an integral homology $D^{2} \times S^{2}$ ). All this follows from the fact that

$$
\begin{aligned}
d_{1 / 2}(M(1(1,0)(1,1))) & =-\frac{3}{2}, \\
d_{-1 / 2}(M(1(1,0)(1,1))) & =-\frac{5}{2},
\end{aligned}
$$


together with Theorem 9.11 of [15].

Lemma 4.7 We have the identification:

$$
H F^{+}(M(1(0,0)(0,0))) \cong\left(\mathcal{T}_{0}^{+}\right)^{6} \oplus\left(\mathcal{T}_{-1}^{+}\right)^{8} \oplus\left(\mathcal{T}_{-2}^{+}\right)^{2} \oplus V_{(0,0),(0,0)}
$$

Moreover, if we let $\mathbb{Z}_{(0)}^{6} \subset \widehat{H F}_{0}(M(1(0,0)(0,0)))$ be a subgroup which maps isomorphically onto

$$
\left(\operatorname{Ker} U \mid\left(\mathcal{T}_{0}^{+}\right)^{6}\right) \subset H F_{0}^{+}(M(1(0,0)(0,0))),
$$

then the map induced by the $H_{1}$-action

$$
H_{1}(M(1(0,0)(0,0)) ; \mathbb{Z}) \otimes \mathbb{Z}_{(0)}^{6} \longrightarrow \widehat{H F}_{-1}(M(1(0,0)(0,0)))
$$

has six-dimensional image.

Proof We find it convenient to work with $\widehat{H F}$. The last isomorphism of the previous lemma shows that

$$
\widehat{H F}(M(1(1,0)(1,1))) \cong \mathbb{Z}_{(-5 / 2)}^{2} \oplus \mathbb{Z}_{(-3 / 2)}^{2} \oplus \widehat{V}_{(1,0),(1,1)} .
$$

Recall (see Section 8 of [15]) that

$$
\begin{aligned}
\widehat{H F}(M(1(\infty, 0),(1,1))) & \cong \mathbb{Z}_{(-3 / 2)} \oplus \mathbb{Z}_{(-5 / 2)} \\
\widehat{H F}(M(1(0,0),(\infty, 1)) & \cong \mathbb{Z}_{(0)}^{2} \oplus \mathbb{Z}_{(-1)}^{2} .
\end{aligned}
$$

Observe that each $Y=M(1(b, c)(d, e))$ has standard $H F^{\infty}$. This gives rise to a subspace of $\widehat{H F}(Y)$ of rank $2^{b_{1}(Y)}$, which comes from the intersection of the image of $H F^{\infty}(Y)$ in $H F^{+}(Y)$ with the kernel of $U$. We denote this space $\widehat{W}$. (Note that we are dropping $b, c, d$, and $e$ from the notation temporarily, as the argument we give here is independent of them). Moreover, there is also a fourdimensional subspace in $\widehat{H F}(Y)$ according to Lemma 4.4, which we denote $\widehat{V}$. We claim that $\widehat{V} \cap \widehat{W}=0$. This follows since (by construction) $\widehat{W}$ injects into $H F^{+}(Y)$ under the natural map $i: \widehat{H F}(Y) \longrightarrow H F^{+}(Y)$, but is in the kernel of the composite of this map with the natural map $\pi: H F^{+}(Y) \longrightarrow H F_{\text {red }}^{+}(Y)$. On the other hand, $\widehat{V}$ consists of one two-dimensional summand which is in the kernel $i$, and another which injects into $H F_{\text {red }}^{+}(Y)$ under the composite $\pi \circ i$. Now since $b_{1}(M(1(b, c)(d, e)))$ is given by the number of zero entries among the $b, c, d, e$, the above observations show that $\widehat{H F}$ of each of these three-manifolds satisfies:

$$
\begin{aligned}
& \operatorname{rk}(\widehat{H F}(M(1(0,1),(1,1)))) \geq 6 \\
& \operatorname{rk}(\widehat{H F}(M(1(0,0),(1,1)))) \geq 8 \\
& \operatorname{rk}(\widehat{H F}(M(1(0,0),(0,1)))) \geq 12
\end{aligned}
$$

Geometry $8 \mathcal{T}$ Topology, Volume 7 (2003) 
There are also two exact sequences, associated to triples:

$$
\begin{array}{lll}
M(1(\infty, 0),(1,1)) \cong-\Sigma(2,3,5) \#\left(S^{2} \times S^{1}\right), & M(1(0,0)(1,1)), & M(1(1,1),(1,1))) \\
M(1(0,0),(\infty, 1)), & M(1(0,0)(0,1)), & M(1(0,0),(0,0))) .
\end{array}
$$

We claim that there are only two possible answers for $\widehat{H F}(M(1(0,0)(0,0)))$ which are consistent with all of these constraints:

$$
\widehat{H F}(M(1(0,0)(0,0))) \cong \mathbb{Z}_{(0)}^{6} \oplus \mathbb{Z}_{(-1)}^{8} \oplus \mathbb{Z}_{(-2)}^{2} \oplus \widehat{V}_{(0,0)(0,0)} .
$$

or

$$
\widehat{H F}(M(1(0,0)(0,0))) \cong \mathbb{Z}_{(0)}^{6} \oplus \mathbb{Z}_{(-1)}^{9} \oplus \mathbb{Z}_{(-2)}^{3} \oplus \widehat{V}_{(0,0)(0,0)} .
$$

Again, as in the proof of Lemma 4.5, we are using here the fact that the various $\widehat{V}_{(b, c)(d, e)}$ are mapped to one another by the corresponding maps, which follows from naturality of the cobordism invariants, together with the fact that the relevant cobordisms connecting the corresponding $M(0(b, c)(d, e))$ all admit genus two Lefschetz fibrations.

The latter case is ruled out as follows. Suppose it is realized. Then, we consider the long exact sequence for the triple

$$
\left(M(\infty(0,0),(0,0)) \cong \#^{4}\left(S^{2} \times S^{1}\right), \quad M(0(0,0)(0,0)) \cong S^{1} \times \Sigma_{2}, \quad M(1(0,0),(0,0))\right) .
$$

In this case, $\widehat{H F}_{3 / 2}\left(S^{1} \times \Sigma_{2}, \mathfrak{t}_{0}\right) \cong \mathbb{Z}$ (it is the image of the top-dimensional generator of $\left.\widehat{H F}\left(\#^{4}\left(S^{2} \times S^{1}\right)\right)\right)$. Now, since $\widehat{H F}_{-3 / 2}\left(S^{1} \times \Sigma_{2}\right)$ surjects onto

$$
\operatorname{Ker}\left(\widehat{H F}_{-2}(M(1(0,0),(0,0))) \cong \mathbb{Z}^{3} \longrightarrow \widehat{H F}_{-2}\left(\#^{4}\left(S^{2} \times S^{1}\right)\right) \cong \mathbb{Z}\right),
$$

it follows that $\operatorname{rk} \widehat{H F}_{-3 / 2}\left(S^{1} \times \Sigma_{2}\right) \geq 2$. But this contradicts the fact that

$$
\widehat{H F}_{k}\left(S^{1} \times \Sigma_{2}\right) \cong \widehat{H F}_{-k}\left(S^{1} \times \Sigma_{2}\right),
$$

which follows from the fact that $S^{1} \times \Sigma_{2}$ admits an orientation-reversing diffeomorphism.

It follows that we have isomorphism from Equation (18), which easily translates to the claimed identification of $\mathrm{HF}^{+}$.

For the claim about the $H_{1}$ action, we investigate the above isomorphisms more carefully. Indeed, we break the verification into pieces, verifying first that the image of

$$
\begin{aligned}
H_{1}(M(1,(0,0),(0, \infty)) ; \mathbb{Z}) \otimes \widehat{H F}_{1 / 2}(M(1,(0,0),(0, \infty))) \\
\longrightarrow \widehat{H F}_{-1 / 2}(M(1(0,0),(0, \infty)))
\end{aligned}
$$


has rank two. But this follows readily from the fact that

$$
M(1(0,0),(0, \infty))=M(1(0,0)) \#\left(S^{2} \times S^{1}\right),
$$

and thus (according to the Künneth principle for connected sums, cf. Proposition 6.1 of [12])

$$
\widehat{H F}\left(M(1(0,0),(0, \infty)) \cong \widehat{H F}(M(1(0,0))) \otimes_{\mathbb{Z}} H^{*}\left(S^{1}\right),\right.
$$

where the homology class supported in the $S^{2} \times S^{1}$ acts as contraction on $H^{*}\left(S^{1}\right)$. In particular, action by this homology class surjects onto the bottomdimensional homology of $\widehat{H F}(M(1(0,0),(0, \infty))$ ) (which in this case is supported in dimension $-1 / 2$ ).

We claim also that the map

$$
\begin{aligned}
H_{1}(M(1,(0,0),(0,1)) ; \mathbb{Z}) \otimes \widehat{H F}_{-1 / 2}(M(1,(0,0),(0,1))) & \\
& \longrightarrow \widehat{H F}_{-3 / 2}(M(1,(0,0),(0,1)))
\end{aligned}
$$

has four-dimensional image. Indeed, we claim that chasing through the above isomorphisms, the natural maps

$$
\begin{array}{rll}
H F_{d}^{\infty}(M(1(0,0),(0,1))) & \longrightarrow & H F_{d}^{+}(M(1(0,0),(0,1))) \\
\widehat{H F}_{d}(M(1(0,0),(0,1))) & \longrightarrow & H F_{d}^{+}(M(1(0,0),(0,1)))
\end{array}
$$

are isomorphisms when $d=-1 / 2,-3 / 2$. Indeed, the above natural maps respect the $H_{1}$-actions. Moreover, since $H F^{\infty}$ of $Y=M(1(0,0),(0,1))$ is standard, and $b_{1}(Y)=3$, we see that if $H=H_{1}(Y ; \mathbb{Z})$, and $H^{*}=H^{1}(Y ; \mathbb{Z})$

$$
\begin{aligned}
& H F_{-1 / 2}^{\infty}(Y) \cong \Lambda^{3} H^{*} \oplus H^{*} \\
& H F_{-3 / 2}^{\infty}(Y) \cong \Lambda^{2} H^{*} \oplus \Lambda^{0} H^{*},
\end{aligned}
$$

where the $H=H_{1}$ action is modelled by contraction. The claim is now immediate.

Finally, the claim of the lemma then follows easily from a glance at the $\widehat{H F}$-long exact sequence for the triple

$$
(M(1,(0,0),(0, \infty)), \quad M(1,(0,0),(0,0)) \quad M(1,(0,0),(0,1))),
$$

bearing in mind that all maps are equivariant under the action of the onedimensional homology, and using the rank calculations established above.

Lemma 4.8 Let $Y$ be a three-manifold with $b_{1}(Y)=5$, and $\mathfrak{t}_{0}$ be a $\operatorname{Spin}^{c}$ structure whose first Chern class is torsion. Then, in each degree $k$,

$$
9 \leq \operatorname{rkH} F_{k}^{\infty}\left(Y, \mathfrak{t}_{0}\right) \leq 16 .
$$


Indeed, when the lower bound is realized, the action

$$
H_{1}(Y ; \mathbb{Z}) \otimes H F_{\mathrm{odd}}^{\infty}\left(Y, \mathfrak{t}_{0}\right) \longrightarrow H F_{\mathrm{ev}}^{\infty}\left(Y, \mathfrak{t}_{0}\right)
$$

is trivial. (Here, we are using the absolute $\mathbb{Z} / 2 \mathbb{Z}$ grading on $H F^{\infty}$, which is characterized by the property that $\underline{H F}_{\mathrm{ev}}^{\infty}$ is non-trivial.)

Proof Now, to estimate $H F_{k}^{\infty}\left(Y, t_{0}\right)$, we use the universal coefficients spectral sequence. The $E_{2}$ term here is a repeating pattern of

$$
\begin{array}{llllll}
\mathbb{Z} & H^{1}(Y) & \Lambda^{2} H^{1}(Y) & \Lambda^{3} H^{1}(Y) & \Lambda^{4} H^{1}(Y) & \Lambda^{5} H^{1}(Y) \\
0 & 0 & 0 & 0 & 0 & 0
\end{array}
$$

(i.e. the repeating pattern comes about by the various $U$ powers). It is easy to see that the total rank of $E_{\infty}$ is minimized if the $d_{3}$ differential restricts as a surjection from $\Lambda^{3} H^{1}(Y) \longrightarrow \mathbb{Z}$, an isomorphism from $\Lambda^{4} H^{1}(Y) \longrightarrow$ $H^{1}(Y)$, and an injection from $\Lambda^{5} H^{1}(Y) \longrightarrow \Lambda^{2} H^{1}(Y)$. In that case, the rank of $H F_{k}\left(Y, \mathfrak{t}_{0}\right)$ (for each $k$ ) is 9 . For such a three-manifold, $H F^{\infty}(Y)$ is a quotient of a $\mathbb{Z}\left[U, U^{-1}\right] \otimes_{\mathbb{Z}} \Lambda^{*} H_{1}(Y ; \mathbb{Z})$-submodule of

$$
\left(\Lambda^{3} H^{1}(Y) \oplus \Lambda^{2} H^{1}(Y)\right) \otimes_{\mathbb{Z}} \mathbb{Z}\left[U, U^{-1}\right] .
$$

In particular, the $H_{1}(Y)$-action on elements of odd parity $\left(\Lambda^{2} H^{1}(Y)\right)$ is trivial. (Recall that the parity is defined so that $\Lambda^{b_{1}} H^{1}(Y)$ has even parity.)

Theorem 4.9 Letting $\mathfrak{t}_{0} \in \operatorname{Spin}^{c}\left(S^{1} \times \Sigma_{2}\right)$ be the $\operatorname{Spin}^{c}$ structure with trivial first Chern class, we have the $\mathbb{Z}[U]$-module identification

$$
H F^{+}\left(S^{1} \times \Sigma_{2}, \mathfrak{t}_{0}\right) \cong\left(\mathcal{T}_{3 / 2}^{+}\right) \oplus\left(\mathcal{T}_{1 / 2}^{+}\right)^{9} \oplus\left(\mathcal{T}_{-1 / 2}^{+}\right)^{9} \oplus\left(\mathcal{T}_{-3 / 2}^{+}\right)
$$

In particular,

$$
\widehat{H F}\left(S^{1} \times \Sigma_{2}, \mathfrak{t}_{0}\right) \cong \mathbb{Z}_{(3 / 2)} \oplus \mathbb{Z}_{(1 / 2)}^{9} \oplus \mathbb{Z}_{(-1 / 2)}^{9} \oplus \mathbb{Z}_{(-3 / 2)} .
$$

Moreover, the only other non-trivial $\mathrm{Spin}^{c}$ structures with non-trivial $\mathrm{HF}^{+}$are the ones with $c_{1}(\mathfrak{t})= \pm \mathrm{PD}\left[S^{1}\right]$ (where here $\left[S^{1}\right]$ represents the fiber factor of $S^{1} \times \Sigma_{2}$ ); for each of those, we have that $H F^{+}$is isomorphic to $\mathbb{Z}$.

Proof Consider the triple:

$\left(M(\infty(0,0),(0,0)) \cong \#^{4}\left(S^{2} \times S^{1}\right), M(0(0,0)(0,0)) \cong S^{1} \times \Sigma_{2}, M(1(0,0),(0,0))\right)$

Observe that the map from $H F^{+}\left(\#^{4}\left(S^{2} \times S^{1}\right)\right)$ to $\bigoplus_{\left\{\mathfrak{t} \mid c_{1}(\mathfrak{t}) \neq 0\right\}} H F^{+}\left(\Sigma_{2} \times S^{1}\right)$ is trivial, and hence, so is the map induced on $\widehat{H F}$. It follows that $\widehat{W}$ maps 
isomorphically onto $\widehat{V}$ in the $\widehat{H F}$ long-exact sequence for the triple which now reads:

$\cdots \stackrel{F_{1}}{\longrightarrow} \widehat{H F}\left(\#^{4}\left(S^{2} \times S^{1}\right)\right) \stackrel{F_{2}}{\longrightarrow} \widehat{H F}\left(S^{1} \times \Sigma_{2}, \mathfrak{t}_{0}\right) \oplus \widehat{W} \stackrel{F_{3}}{\longrightarrow} \mathbb{Z}_{(0)}^{6} \oplus \mathbb{Z}_{(-1)}^{8} \oplus \mathbb{Z}_{(-2)}^{2} \oplus \widehat{V} \rightarrow \cdots$

Of course, as a graded group, we have that

$$
\widehat{H F}\left(\#^{4}\left(S^{2} \times S^{1}\right)\right) \cong \mathbb{Z}_{(2)} \oplus \mathbb{Z}_{(1)}^{4} \oplus \mathbb{Z}_{(0)}^{6} \oplus \mathbb{Z}_{(-1)}^{4} \oplus \mathbb{Z}_{(-2)} .
$$

Now, let $C$ denote the rank of the kernel of the map

$$
F_{3}: \widehat{H F}_{0}(M(1(0,0),(0,0))) \cong \mathbb{Z}^{6} \longrightarrow \widehat{H F}_{0}\left(\#^{4}\left(S^{2} \times S^{1}\right)\right) \cong \mathbb{Z}^{6} .
$$

Since $\widehat{H F}_{k}\left(S^{1} \times \Sigma_{2}\right) \cong \widehat{H F}_{-k}\left(S^{1} \times \Sigma_{2}\right)$ (which in turn follows from the fact that the three-manifold has an orientation-reversing diffeomorphism), it follows that

$$
\widehat{H F}\left(S^{1} \times \Sigma_{2}, \mathfrak{t}_{0}\right)=\mathbb{Z}_{(3 / 2)} \oplus \mathbb{Z}_{(1 / 2)}^{4+C} \oplus \mathbb{Z}_{(-1 / 2)}^{4+C} \oplus \mathbb{Z}_{(-3 / 2)} .
$$

Now, it follows from Lemma 4.8 that $C \geq 4$.

The case where $C=6$ is excluded by the $H_{1}$ action as follows. We have seen that the map

$$
F_{2}: \widehat{H F}_{-1}(M(1(0,0),(0,0))) \longrightarrow \widehat{H F}_{-1}\left(\#^{4}\left(S^{1} \times S^{2}\right)\right)
$$

is surjective; it follows that there must be some element in the six-dimensional subspace of $\widehat{H F}_{-1}(M(1(0,0),(0,0)))$ which is the $H_{1}$-image of $\widehat{H F}_{0}$ with nonzero projection under $F_{2}$. But by the naturality of the $H_{1}$ action, such an element must have zero image under $F_{2}$, since $C=6$ is the hypothesis that

$$
F_{2}: \widehat{H F}_{0}(M(1(0,0),(0,0))) \longrightarrow \widehat{H F}_{0}\left(\#^{4}\left(S^{1} \times S^{2}\right)\right)
$$

is identically zero.

To rule out the case where $C=4$, we proceed as follows. If $C=4$, then the lower bound on the rank of $H F^{\infty}$ Lemma 4.8 is realized. On the other hand, the image of the top-dimensional class in $\widehat{H F}\left(\#^{4}\left(S^{1} \times S^{2}\right)\right)$ has odd parity in $\widehat{H F}\left(S^{1} \times \Sigma_{2}\right)$, and yet it has non-trivial images under the $H_{1}$ action, contradicting that lemma.

The only remaining case is $C=5$ in Equation (19). This easily translates to the claimed identification of $\mathrm{HF}^{+}$. 


\subsection{Further speculation}

Although $\mathrm{HF}^{+}$of a three-manifold is a subtle invariant, we know that $H F^{\infty}$ is not: it remains unchanged under integral surgeries which preserve $b_{1}$. Still, it is useful to know $H F^{\infty}$ as a starting point for calculations of $\mathrm{HF}^{+}$.

As a computational tool, we have a spectral sequence whose $E_{2}$ term is given by

$$
\mathbb{Z}\left[U, U^{-1}\right] \otimes_{\mathbb{Z}} \Lambda^{*} H^{1}(Y ; \mathbb{Z}),
$$

which converges to $H F^{\infty}(Y)$. Thus a three manifold $Y$ has standard $H F^{\infty}$ if all the differentials $d_{i}$ for $i \geq 2$ are trivial.

We have seen (cf. Proposition 8.4 of [15]) that $T^{3}$ is a three-manifold whose $H F^{\infty}$ is not standard. In fact, Theorem 4.9 provides us with another such three-manifold: in each dimension, the rank of $H F^{\infty}$ (as a $\mathbb{Z}$-module) is only ten, rather than sixteen. It is natural to expect that the cohomology ring of $Y$ plays an important role here. More concretely, we make the following conjecture (which is easily seen to be consistent with the above calculations):

Conjecture 4.10 Let $Y$ be a closed, oriented three-manifold equipped with a torsion $\operatorname{Spin}^{c}$ structure $\mathfrak{t}_{0}$. The spectral sequence for $H F^{\infty}\left(Y, \mathfrak{t}_{0}\right)$ collapses after the $E_{3}$ stage, and moreover the differential

$$
d_{3}: \Lambda^{i} H^{1}(Y ; \mathbb{Z}) \otimes_{\mathbb{Z}} U^{j} \longrightarrow \Lambda^{i-3} H^{1}(Y ; \mathbb{Z}) \otimes_{\mathbb{Z}} U^{j-1}
$$

is given by the homological pairing:

$d_{3}\left(\phi_{1} \wedge \ldots \wedge \phi_{i}\right)=\frac{1}{3 ! \cdot(i-3) !} \sum_{\sigma \in \mathfrak{S}_{i}}(-1)^{\sigma}\left\langle\phi_{\sigma(1)} \cup \phi_{\sigma(2)} \cup \phi_{\sigma(3)},[Y]\right\rangle \cdot \phi_{\sigma(4)} \wedge \ldots \wedge \phi_{\sigma(i)}$,

where $\mathfrak{S}_{i}$ denotes the permutation group on $i$ letters, and $(-1)^{\sigma}$ denotes the sign of the permutation.

\section{References}

[1] S K Donaldson, Floer homology groups in Yang-Mills theory, volume 147 of Cambridge Tracts in Mathematics, Cambridge University Press (2002), with the assistance of M Furuta and D Kotschick

[2] N D Elkies, A characterization of the $Z^{n}$ lattice, Math. Res. Lett. 2 (1995) $321-326$

[3] R Fintushel, R J Stern, Instanton homology of Seifert fibered homology three spheres, Proc. of the London Math. Soc. 61 (1990) 109-137 
[4] K A Frøyshov, The Seiberg-Witten equations and four-manifolds with boundary, Math. Res. Lett 3 (1996) 373-390

[5] KA Frøyshov, An inequality for the h-invariant in instanton Floer theory (2001), arXiv:math.DG/0111038

[6] R E Gompf, A I Stipsicz, 4-manifolds and Kirby calculus, volume 20 of Graduate Studies in Mathematics, American Mathematical Society (1999)

[7] P A Kirk, E P Klassen, Representation spaces of Seifert fibered homology spheres, Topology 30 (1991) 77-95

[8] P B Kronheimer, T S Mrowka, The genus of embedded surfaces in the projective plane, Math. Research Letters 1 (1994) 797-808

[9] J W Morgan, The Seiberg-Witten Equations and Applications to the Topology of Smooth Four-Manifold, Mathematical Notes 44, Princeton University Press (1996)

[10] TS Mrowka, P S Ozsváth, B Yu, Seiberg-Witten Monopoles on Seifert Fibered Spaces, Comm. in Analysis and Geometry 5 (1997) 685-793

[11] P S Ozsváth, Z Szabó, Holomorphic triangles and invariants for smooth fourmanifolds, arXiv:math.SG/0110169

[12] P S Ozsváth, Z Szabó, Holomorphic disks and three-manifold invariants: properties and applications (2001), arXiv:math.SG/0105202, to appear in Annals of Math.

[13] P S Ozsváth, Z Szabó, Holomorphic disks and topological invariants for closed three-manifolds (2001), arXiv:math.SG/0101206, to appear in Annals of Math.

[14] P S Ozsváth, Z Szabó, Holomorphic disk invariants for symplectic fourmanifolds (2002), arXiv:math.SG/0201059

[15] P S Ozsváth, Z Szabó, Absolutely graded Floer homologies and intersection forms for four-manifolds with boundary, Advances in Mathematics 173 (2003) 179-261

[16] P S Ozsváth, Z Szabó, On Heegaard Floer homology and Seifert fibered surgeries (2003), arXiv:math.GT/0301026

[17] E Witten, Monopoles and Four-Manifolds, Math. Research Letters 1 (1994) 769-796 